\title{
In-depth assembly of organ and development dissected Picrorhiza kurroa proteome map using mass spectrometry
}

Manglesh Kumari 1,2, Upendra Kumar Pradhan 1,2,3,4, Robin Joshi ${ }^{1,2}$, Ashwani Punia 1,2, Ravi Shankar ${ }^{1,2,3}$ and Rajiv $\operatorname{Kumar}^{1,2^{*}}$ (D)

\begin{abstract}
Background: Picrorhiza kurroa Royle ex Benth. being a rich source of phytochemicals, is a promising high altitude medicinal herb of Himalaya. The medicinal potential is attributed to picrosides i.e. iridoid glycosides, which synthesized in organ-specific manner through highly complex pathways. Here, we present a large-scale proteome reference map of $P$. kurroa, consisting of four morphologically differentiated organs and two developmental stages.

Results: We were able to identify 5186 protein accessions (FDR $<1 \%$ ) providing a deep coverage of protein abundance array, spanning around six orders of magnitude. Most of the identified proteins are associated with metabolic processes, response to abiotic stimuli and cellular processes. Organ specific sub-proteomes highlights organ specialized functions that would offer insights to explore tissue profile for specific protein classes. With reference to P. kurroa development, vegetative phase is enriched with growth related processes, however generative phase harvests more energy in secondary metabolic pathways. Furthermore, stress-responsive proteins, RNA binding proteins (RBPs) and post-translational modifications (PTMs), particularly phosphorylation and ADP-ribosylation play an important role in P. kurroa adaptation to alpine environment. The proteins involved in the synthesis of secondary metabolites are well represented in $P$. kurroa proteome. The phytochemical analysis revealed that marker compounds were highly accumulated in rhizome and overall, during the late stage of development.
\end{abstract}

Conclusions: This report represents first extensive proteomic description of organ and developmental dissected $P$. kurroa, providing a platform for future studies related to stress tolerance and medical applications.

Keywords: Picrorhiza kurroa, Proteome, PTMs, RBPs, Picrosides, Metabolites

\section{Background}

The Himalaya is an important repository of medicinal and aromatic plants that are a rich source of novel compounds for pharmaceutical industry. Most of them are confined to high-altitude regions that are characterized by extremes temperatures, radiations, drought, snow cover and partial pressure of gases. These plant species

\footnotetext{
*Correspondence: rajiv@ihbt.res.in

${ }^{1}$ Biotechnology Division, CSIR-Institute of Himalayan Bioresource

Technology (IHBT), Palampur 176061, HP, India

Full list of author information is available at the end of the article
}

synthesize extensive arrays of secondary metabolites having medicinal importance. Owing to the successful acclimatization in extreme environments, high-altitude plants can serve as a model for elucidation of combinatorial stress tolerance mechanisms. Picrorhiza kurroa Royle ex Benth. (Family Plantaginaceae), a perennial herb of Himalaya (3000-5000 masl), has been used in traditional and modern medicine systems as hepatoprotective, antiperiodic, cholagouge, stomachic, antiamoebic, antioxidant, antihelmintic, antiinflammatory, cardiotonic, laxative, carminative and expectorant [1-3]. The 
medicinal potential is mostly ascribed to iridoid glycosides i.e. picrosides, which are used in herbal formulation of more than 2000 drugs [1, 4]. Morphologically, plants are herbaceous with an elongated rhizome (rootstock) and aerial portion consists of basal leaves and terminal inflorescence (flowering spike) [5]. P. kurroa propagates vegetatively as young buds on rhizomes, which subsequently develops as a new rhizome with autonomous root and shoot sections [6]. In addition, rhizome survives underground during snow cover whereas, shoot emerges rapidly during onset of suitable growth conditions. P. kurroa synthesizes active phytochemicals in tissue/organ-specific manner through highly complex biosynthetic pathways [7]. Due to active phytochemical ingredients, the global annual demand of this plant is increased to 500 tons, whereas its supply is only 375 tons $[1,8]$. Consequently, proteomic technologies provide one of the best choices for the functional analysis of translated parts of the genome. In general, plant proteome studies have increased over the past few years, only the model organisms and food crops continue to be studied. Since, proteins can be better conserved, the identification of non-model protein accessions by comparing them to familiar orthologous proteins would result into functional identification across different species [9]. This cross species identification of proteins is the best approach for extensive study of gene products, wherever genome is uncharacterized [10]. The establishment of species-specific proteome maps using multidimensional fractionations of various cell, tissue or organ types has been essential for better understanding of underlying molecular basis of the plant system.

The proteome reference map has been established for a variety of cells, tissues, organs and organelles from non-model plant species such as Miscanthus sinensis, Pisum sativum, Gingo biloba, Triticum aestivum, Arachis hypogaea, Quercus ilex, Pinus radiata, Tectona grandis to model plants like Arabidopsis thaliana, Oryza sativa, Glycine max, Solanum lycopersicum, Medicago truncatula and Zea mays based upon the two-dimensional gel electrophoresis (2D-GE) due to its better resolving power [11-25]. Recently, alternative approaches such as gel-free techniques have also been utilized for the construction of proteome map in Triticum aestivum and Arabidopsis thaliana [26, 27]. However, bottom-up, gelfree approaches are reported less-efficient in non-model systems due to loosing connectivity between proteinderived peptides [28]. Furthermore, taking advantage of one-dimensional gel electrophoresis (1D-GE) coupled with liquid chromatography (LC) prior to mass spectrometry (MS), a high density Arabidopsis proteome was assembled [29]. The key benefit of this combinatorial approach is to facilitate the use of SDS in 1D-GE that ensures solubility of proteins during size separation and reduction in sample complexity before LC, which increases the probability of identifying low abundant proteins [30]. Previous reports have also compared 1D-GE to other pre-fractionation approaches such as isoelectric focusing, strong cation exchange chromatography and protein reversed chromatography at peptide and protein level, which revealed that $1 \mathrm{D}-\mathrm{GE}$ is most effective prefractionation method for greater proteome coverage [31, 32].

Here, we provided 1D-GE-nanoLC-MS/MS based proteomic datasets, which collectively assemble first proteome map of $P$. kurroa covering all four major organs and two developmental stages. Together or separately, these organs function in response to chronic environmental fluctuations at high altitudes. This study has entitled the dissection of developmental and organ-specific proteomes, pathway-centric comparison with functional differences as well as uncovered the protein/enzyme basis of specialized metabolites accumulation in different organs. Further, the identification of stress-responsive proteins, various PTMs and RBPs would provide insights into stress tolerance potential of $P$. kurroa.

\section{Results \\ Overview of organ and developmental dissection of $P$. kurroa proteome}

An illustration of the entire workflow was represented in Fig. 1A. Root, shoot and rhizome were harvested from both early (vegetative/before flowering) and late (reproductive/after flowering) stage, whereas inflorescence was collected from various plants at late stage, which represent seven samples (Fig. 1B). To reduce the impact of biological variation, each of these samples were pool of at least eight different plants. To fractionate proteins, SDS-PAGE from seven different samples of $P$. kurroa was performed. The signal pattern observed inCoomassie brilliant blue (CBB) stained 1D gel was found to be dissimilar for all major organs (Fig. 1C). Although conventional Coomassie staining is less sensitive as compared to silver staining, but quite more simple, suitable and quantitative for downstream applications. Each sample lane was split into eight fractions, which were trypsinized and subsequently analyzed using nanoLC coupled with Agilent 6560 Q-TOF mass spectrometer. The resulted tandem mass spectra were searched against NCBI protein database (2018) containing predicted proteins of all green plants and generated a total of 5186 non-redundant protein accessions (FDR $<1 \%$, peptide score $\geq 3$ and SPI $(\%) \geq 60)$ (Table S1). The proteins having at least one distinct peptide and clustering of similar protein based on sequence homology into same group were considered as positive identification [33]. 


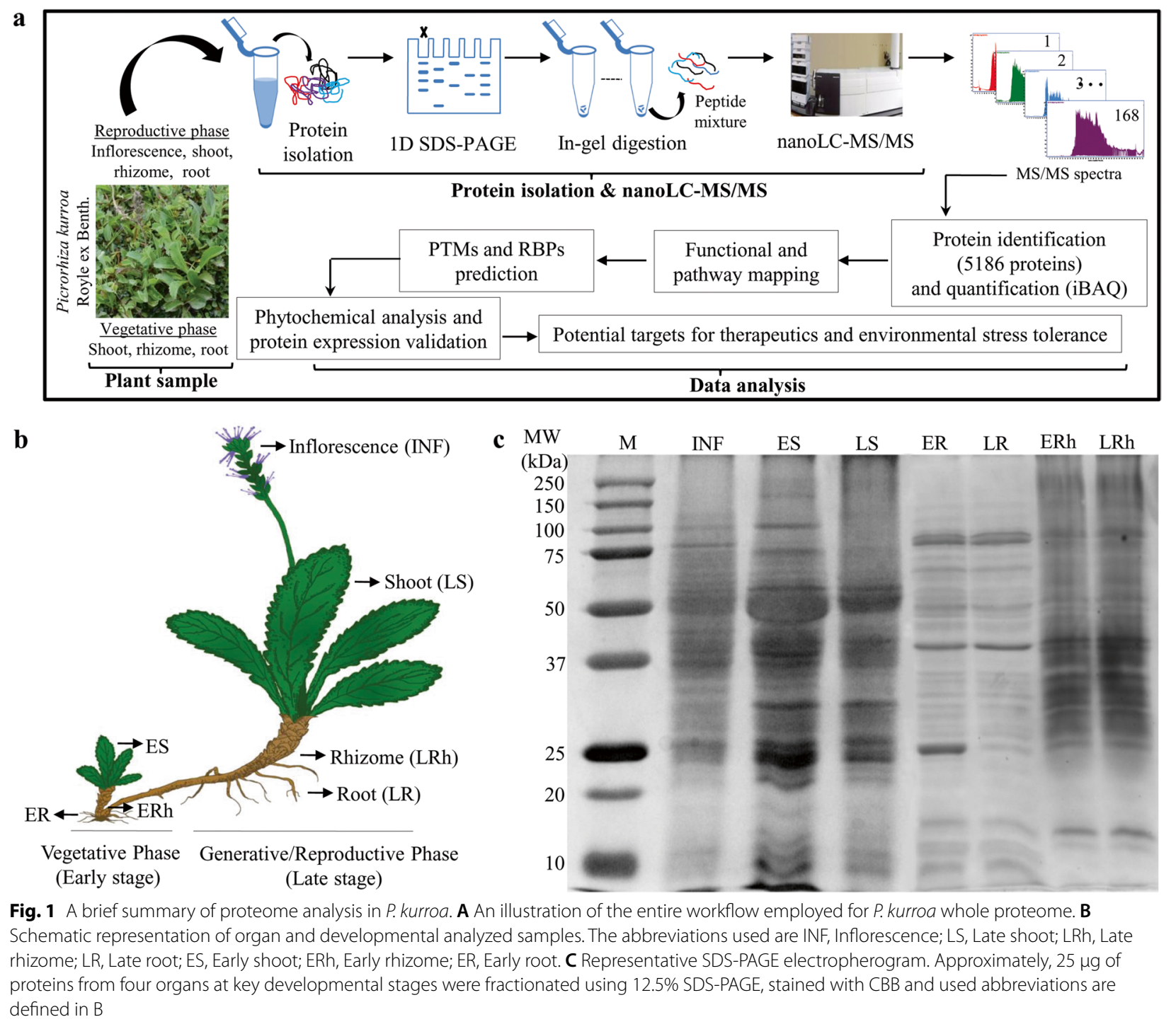

A summary of matched spectra, distinct peptides and proteins identified are displayed in Table 1. On average, the whole proteome ranging from a minimum of 793 protein accessions in root to a maximum of 2267 protein accessions in shoot indicates $P$. kurroa shoot possesses the highest, while root having lowest protein content (Table 1; Table S1). These results are in agreement with our previous report that suggests the pattern of total protein accumulation is organ-specific; shoot/leaf have highest, while root have lowest crude protein content in $P$. kurroa [7]. We provided a circular proteome map of protein abundance in P. kurroa to describe the similarities and differences among organ sub-proteomes (Fig. 2A). This displayed protein groups overlapped among diverse organs in addition to explicitly found in only one organ. Next, the depth of our P. kurroa proteome was assessed by a critical figure of dynamic range (Fig. 2B). The whole proteome dynamic range spanned around six orders of magnitude, when measured based upon protein abundance (iFOT). Similar dynamic ranges of protein expression have also been reported in higher eukaryotes like yeast and Populus that were directed towards deep proteome coverage $[34,35]$.

The raw files were searched against green plant database (NCBInr). The identified proteins (5186) comprise from nine plants species viz. Zea mays (27.76\%), Glycine max (26.41\%), Arabidopsis thaliana (21.63\%), Solanum tuberosum (17.62\%), Triticum aestivum (3.97\%), Nicotiana tabacum (1.13\%), Pisum sativum (0.67\%), Oryza sativa $(0.56 \%)$ and Hordeum vulgare $(0.21 \%)$. Therefore, we performed gene ontology (GO) enrichment of total proteins compared with a customized reference 
Table 1 An outline of the number of matched spectra, distinct peptides and identified proteins through 1D-GE-nanoLC-MS/MS in P. kurroa. Different alphabets represent statistically significant values $(p<0.05)$ as determined by one-way ANOVA followed by Tukey's HSD post-hoc test for each parameter individually in R v. 4.0.0

\begin{tabular}{llll}
\hline Plant organ & Spectra & Distinct Peptides & Proteins \\
\hline Inflorescence & $8107^{\mathrm{b}}$ & $1495^{\mathrm{c}}$ & $1348^{\mathrm{c}}$ \\
Shoot & $10749^{\mathrm{a}}$ & $2531^{\mathrm{a}}$ & $2267^{\mathrm{a}}$ \\
$\quad$ Early shoot & $4837^{\mathrm{de}}$ & $1184^{\mathrm{d}}$ & $1097^{\mathrm{d}}$ \\
$\quad$ Late shoot & $8242^{\mathrm{b}}$ & $1596^{\mathrm{c}}$ & $1433^{\mathrm{c}}$ \\
Rhizome & $10536^{\mathrm{a}}$ & $2070^{\mathrm{b}}$ & $1837^{\mathrm{b}}$ \\
$\quad$ Early rhizome & $5095^{\mathrm{d}}$ & $1081^{\mathrm{d}}$ & $970^{\mathrm{de}}$ \\
$\quad$ Late rhizome & $6990^{\mathrm{c}}$ & $1199^{\mathrm{d}}$ & $1081^{\mathrm{d}}$ \\
Root & $4920^{\mathrm{de}}$ & $841^{\mathrm{e}}$ & $793^{\mathrm{ef}}$ \\
$\quad$ Early Root & $4385^{\mathrm{e}}$ & $678^{\mathrm{ef}}$ & $642^{\mathrm{f}}$ \\
$\quad$ Late Root & $3175^{\mathrm{f}}$ & $490^{\mathrm{f}}$ & $396^{\mathrm{g}}$ \\
Total & $\mathbf{2 5 , 0 6 9}$ & $\mathbf{5 9 2 2}$ & $\mathbf{5 1 8 6}$ \\
\hline
\end{tabular}

database of aforementioned nine plant species using Fisher's exact test to determine the significance of over or under-represented GO terms. This analysis revealed a total of 359 enriched GO terms that were classified into cellular component, molecular function and biological process (Table S3). The five topmost enriched biological process/GO categories include single-organism cellular process, response to abiotic stimulus, single-organism metabolic process, small molecule metabolic process and anatomical structure development (Fig. 2C). However, the under-represented biological process/GO categories include proteins involved in abscisic acid catabolic process, induced systemic resistance, positive regulation of seed germination and gibberellic acid mediated signaling pathway (Fig. 2C). The most enriched molecular functions comprise purine ribonucleoside triphosphate binding, ATP binding, hydrolase activity, while cell periphery, cytoplasm and chloroplast were found to be highly enriched cellular components (Table S3).

\section{Comprehensive map of $P$. kurroa organ proteomes}

A functional view of $P$. kurroa sub-proteomes was generated to compare the GO category enrichment of each organ (Fig. 3A; Table S3). Most of the enriched GO terms were organ-specific, whereas single-organism process was over-represented in all organs. With reference to specific organs, single-organism metabolic process and response to abiotic stimulus were highly enriched in inflorescence. In rhizome, proteins were over-represented for small molecule metabolic process, DNA integration, response to abiotic stimulus, response to oxygen-containing compounds, cell division and transmembrane transport. The most abundant categories in shoot include response to red/far-red light, cellular component organization, glycine metabolic process, regulation of biological quality, photomorphogenesis and mRNA modification, beside others. In contrast, proteins were enriched for developmental process, multicellular organismal process, anatomical structure development, macromolecule modification, transport and localization in root. Thus, each $P$. kurroa organ can be assigned a specific proteome, which reflects organ specialized functions. Further, we exemplify this using principal component analysis (PCA) based on protein abundance in each organ. The first two principal components (Dim 1 and Dim 2) explained $77.4 \%$ of variation and their plotting allocate the clear separation of all organs (Fig. 3B). Similarly, the pairwise correlation value of organ proteomes ranges from a minimum of 0.16 between shoot and inflorescence to a maximum of 0.38 between root and inflorescence (Fig. 3C). The weak correlation coefficients among $P$. kurroa organs indicate the organ specialization, which is reflected by specific types of protein abundance.

Hierarchical cluster analysis was applied to 650 protein accessions (those shared by organs), which resulted in five protein clusters suggests functions of $P$. kurroa organs are distinct (Fig. 4). Across the observed five clusters, the number of protein species ranged from 41 in cluster 5 to 194 in cluster 3 (Table S4). Cluster 1 seemed to be associated with protein abundance in inflorescence and rhizome, whereas cluster 2 comprised of more abundant proteins in aerial portion of P. kurroa i.e. inflorescence and shoot. Cluster 3, the largest one connected with predominant proteins in shoot and rhizome, which showed characteristic biological organization. The protein groups belonging to cluster 4 are found to be most abundant in root and fairly in rhizome, however the smallest cluster 5 showed protein abundance in root and a little bit in shoot.

\section{Pathway analysis between early and late developmental stages}

To visualize the metabolic differences between early and late stages, protein groups having assigned functions (Enzyme EC number) were mapped into KEGG derived metabolic pathways using iPATH (v.3.0; Fig. 5A-B). In addition, pathway enrichment in comparison with aforementioned nine plant species could provide a functional view and thus, expand the biological information regarding $P$. kurroa developmental stages (Fig. 5C). Vegetative phase was found to be significantly enriched for photosynthesis, Calvin cycle, biosynthesis of amino acids, TCA cycle, pentose phosphate pathway, fatty acid degradation, phosphatidylinositol signaling, $\mathrm{ABC}$ transporters, Cys biosynthesis, Val, Leu, Ile degradation and S-adenosyl-L-methionine 


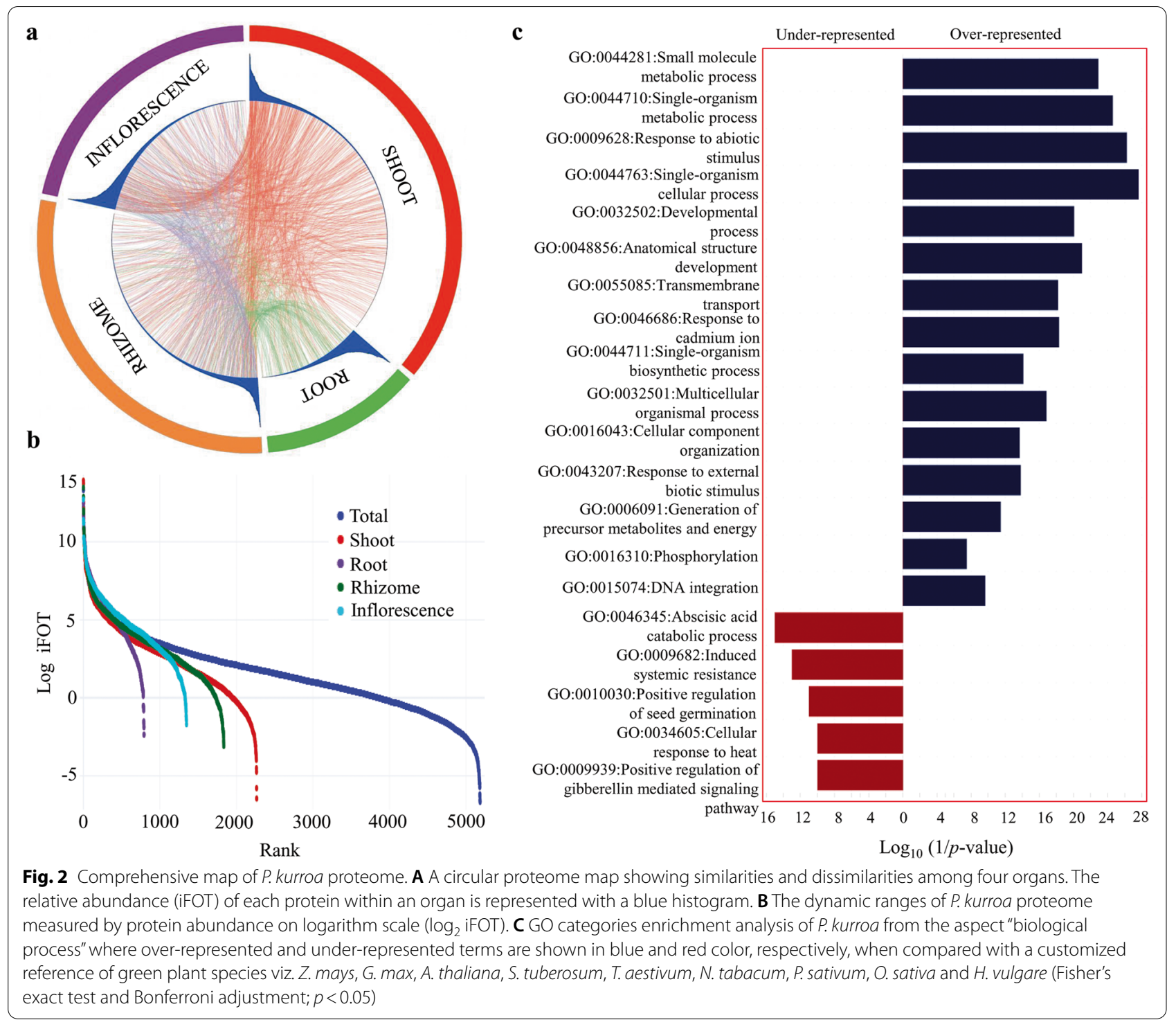

cycle. Relative to the vegetative phase, diverse metabolic pathways such as carbon metabolism, glyoxylate and dicarboxylate metabolism, starch and sucrose metabolism, Gly, Ser and Thr metabolism were found to be enriched in reproductive phase. In addition, processes involved in the production of secondary metabolites like secologanin, strictosidine, GDP-L-fucose and flavonoids besides seed development were significantly enriched in the reproductive phase. Furthermore, proteins linked with biotin metabolism, $\beta$-alanine metabolism, zeatin biosynthesis, taurine and hypotaurine metabolism are exclusively observed in the reproductive stage of $P$. kurroa.

\section{Proteome-wide prediction of RNA binding proteins (RBPs)} and post-translational modifications (PTMs)

The RBPs repertoire responds to a magnitude of biological and environmental cues. The plants RBPs have been less studied compared to other organisms, however studies on A. thaliana and O. sativa showed that many of the RBPs are unique to plants, which suggested their plantspecific functions [36]. In the recent past, it has been well established that besides their role in various developmental processes, they are also essential for plant adaptation to various unfavorable environmental conditions [37]. To identify putative RBPs in P. kurroa proteome, domain analysis was performed for proteins that harbor classical, non-classical or unknown RNA-binding domains (RBDs) reported so far in plants [38]. A total of 1272 putative $P$. kurroa RBPs with 586 RBDs were identified that represents $24.5 \%$ of the whole proteome (Table S5). Most of the putative $P$. kurroa RBPs comprised of protein kinase (Pkinase), leucine-rich repeat (LRR_8), LRR N-terminal (LRRNT_2), pentatricopeptide repeat (PPR; PPR_2), tyrosine kinase (Pkinase_Tyr), nucleotide-binding 


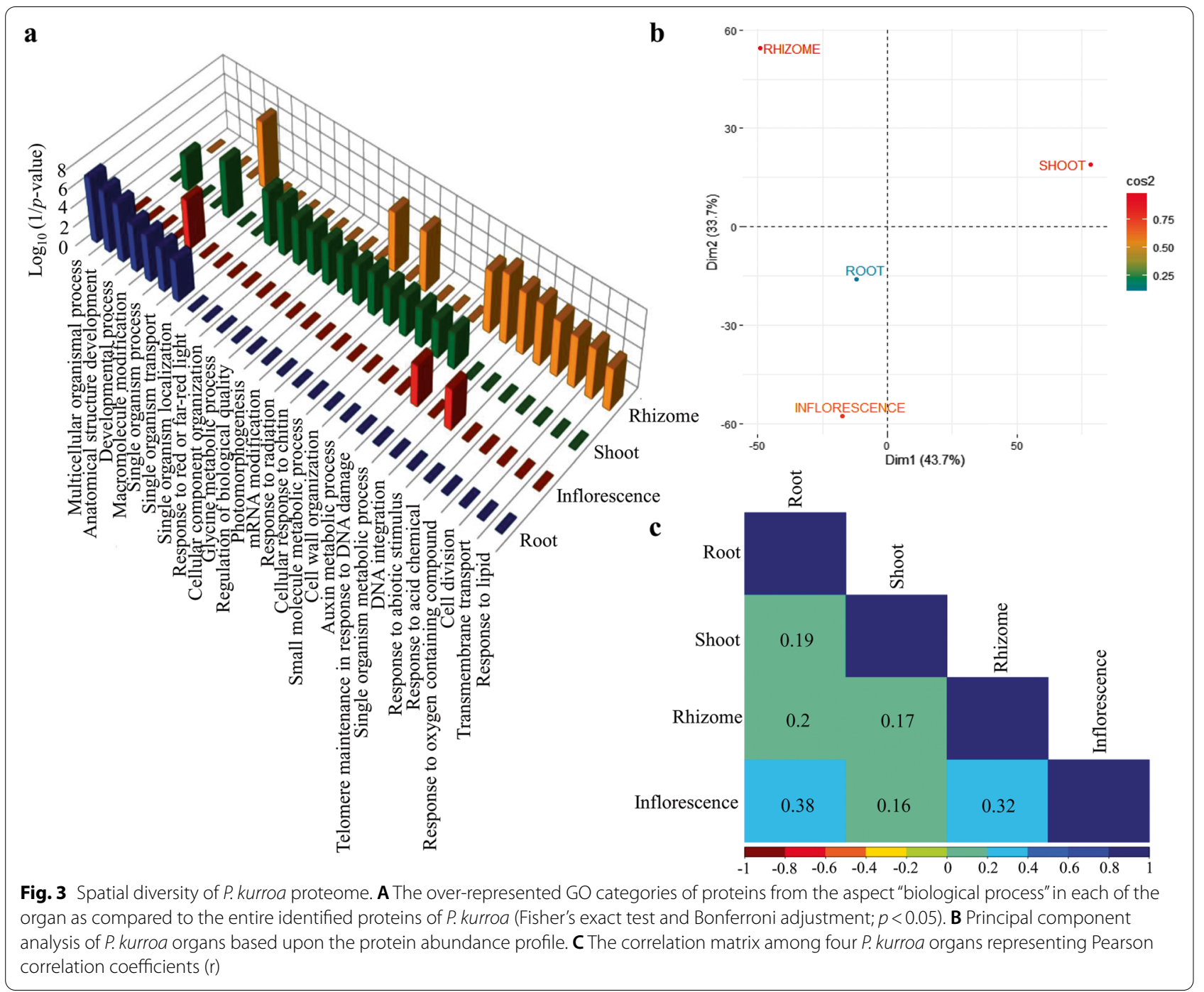

(NB-ARC), cytochrome P450 (p450) and ABC transporter (ABC_tran) domains, which reflect their key role in gene regulation, phosphorylation, protein-protein interaction, synthesis of secondary metabolites, transmembrane translocator and molecular switch for cycling between ADP and ATP bound forms.

Next, we validate $P$. kurroa putative RBPs for their characteristic features by analyzing the physicochemical properties of RBPs in comparison with non-RBPs. Both putative RBP and non-RBP groups span the full array of protein length and abundance with some propensity in the direction of larger length and higher abundance for RBPs compared with non-RBPs (Fig. 6AB) reflecting similar observations as reported previously [38]. Moreover, putative RBPs significantly shifted towards an increased proportion of residues in disordered regions and lower hydrophobicity (Fig. 6C-D) as reported earlier in Arabidopsis [38]. Thus, P. kurroa putative RBPs displayed the biophysical properties anticipated of bonafide RBPs except for the isoelectric point (Fig. 6E). In accordance, we also found thirty-six enriched amino acid motifs of putative RBPs generally comprised of disordered sequences (Fig. 6F; Table S5). Most of these conserved sequence motifs belong to proteins/enzymes/domains that harbor kinase or phosphatase activity, intracellular trafficking, localization signals, ubiquitin conjugation, protein-protein interaction with regulatory proteins, nucleotide triphosphate binding and RNA helicase activity. Overall, P. kurroa putative RBPs also showed significant biases in physicochemical features.

In order to gain structural and functional information of proteins, PTMs were predicted for the total proteome data set using ModPred (Table S6). Notably, phosphorylation (S, T \& Y) and ADP-ribosylation (R) were detected at approximately 1.5 and $1.2 \%$ of total 


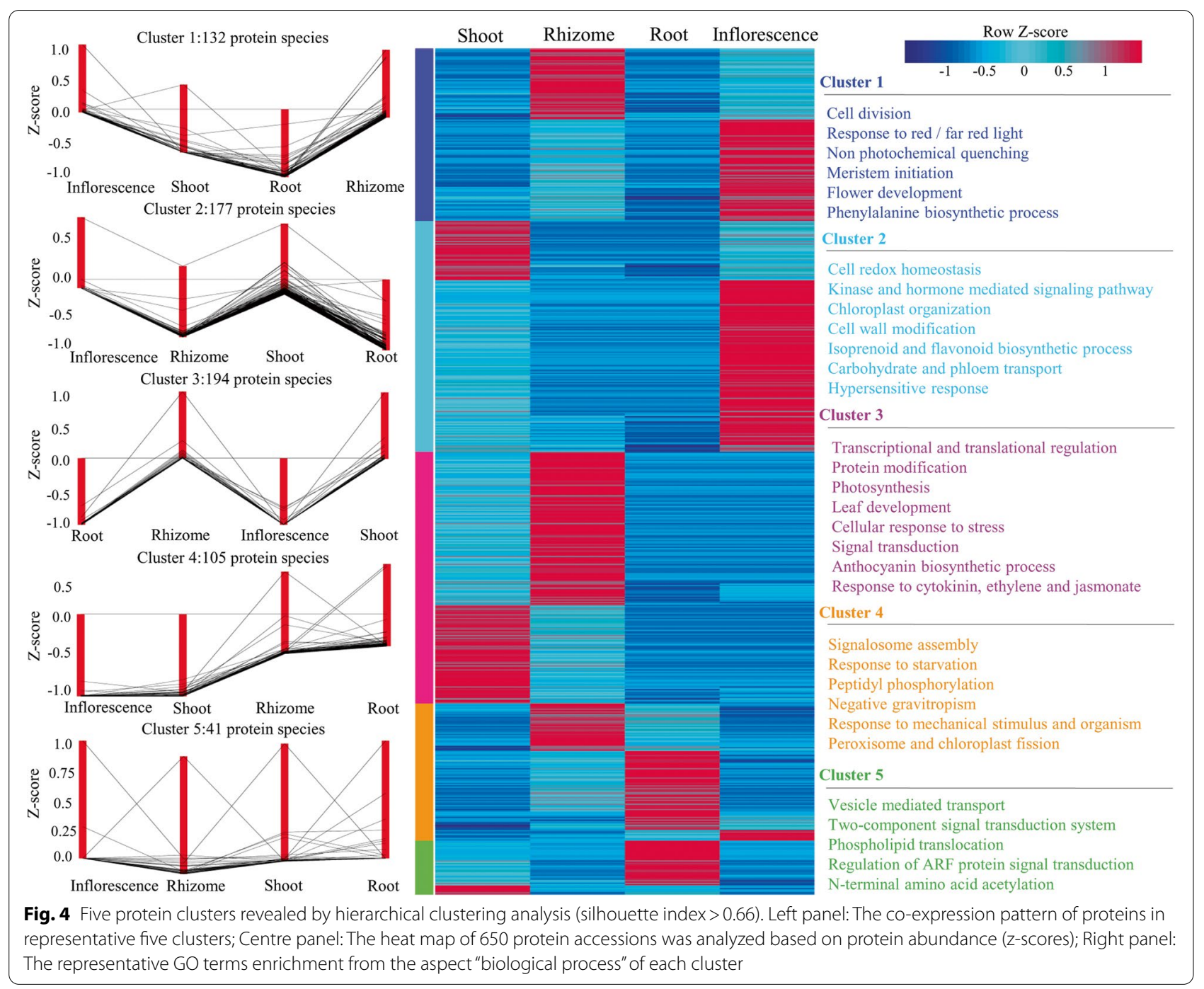

PTMs at peptide level, respectively suggesting their key role in $P$. kurroa developmental and adaptation processes. Further, domain analysis also highlighted Pkinase to be the most enriched domain in whole proteome dataset (Table S5). In addition to the above mentioned PTMs, P. kurroa proteome also demonstrated a significant number of additional PTMs such as carboxylation, ubiquitination, methylation, acetylation and SUMOylation (Table S6).

\section{Discussion}

In order to determine protein atlas of $P$. kurroa, morphologically differentiated four major organs at two developmental stages were chosen for 1D-GE that have the advantage of protein solubilization during fractionation prior to nanoLC coupled with MS for deeper proteome coverage. We used phenol-based protocol for efficient protein extraction that has potential to generate highly pure samples as other plant compounds partition into a discrete aqueous phase from protein-enriched phenol phase. The top ranked proteins detected in P. kurroa total proteome include rubisco large subunit (chloroplast) and ribulose bisphosphate carboxylase (Rubisco). Other most abundant, highly ranked proteins comprised serine carboxypeptidase 45-like, pentatricopeptide repeat-containing protein At1g69290-like, rubisco activase, uncharacterized LOC100305517, glutathione S-transferase tau 7 and ATP synthase CF1 beta subunit (Table S2). In agreement with the depleted GO terms in P. kurroa (Fig. 2C), seed dormancy is common in alpine plants to increase the adaptability towards unfavorable environment. Additionally, low seed fertility in rhizomatous plants developed for their asexual/vegetative reproduction. The plant hormones, predominantly gibberellin and abscisic acid are the foremost endogenous determinants of seed germination and dormancy by acting antagonistically; 

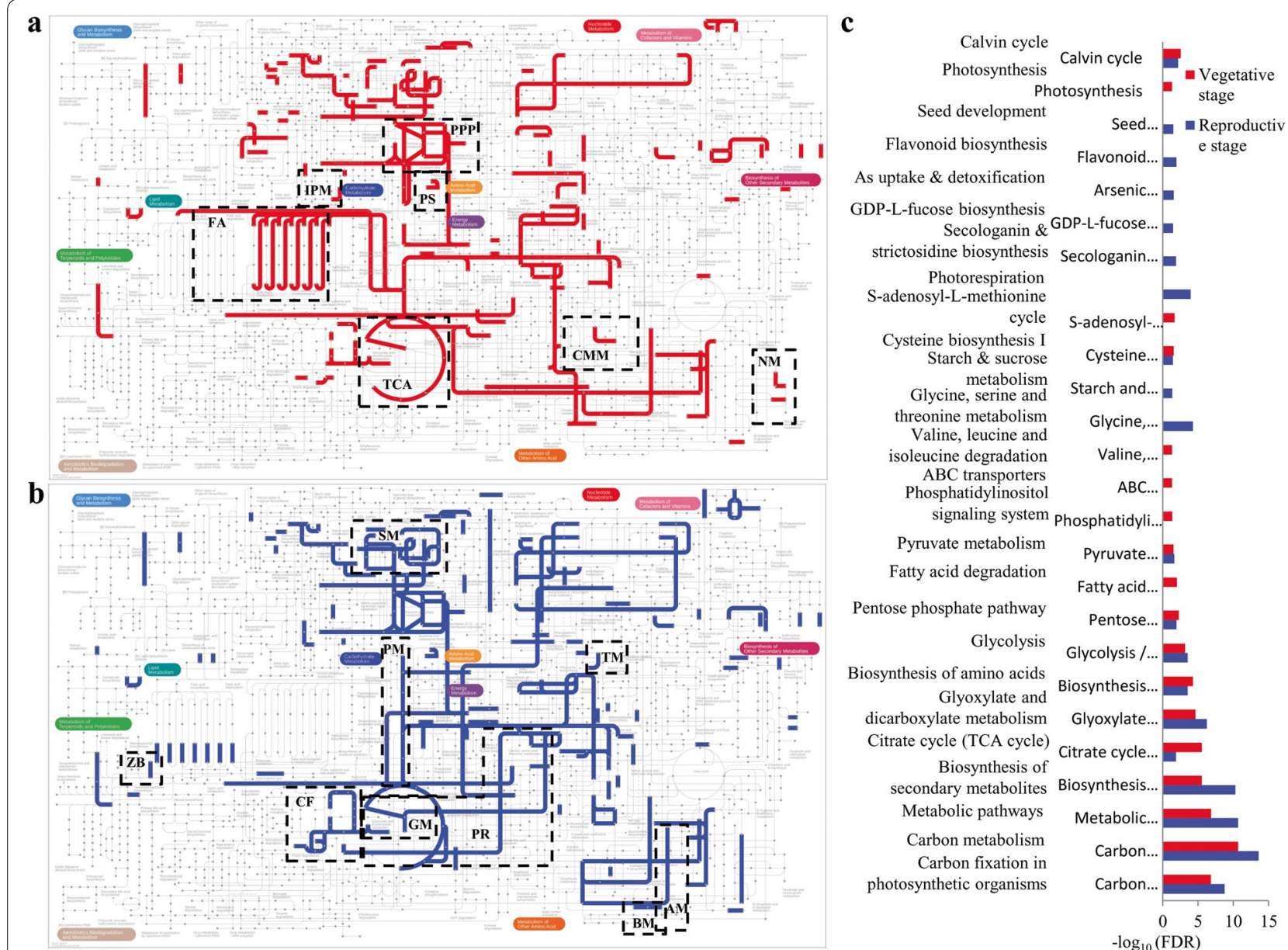

Fig. 5 Pathway-centric comparison of P. kurroa developmental stages. Early and late stages protein groups with assigned functions (Enzyme EC number) were mapped to KEGG pathways using iPath v. 3.0. A Vegetative/early stage and $\mathbf{B}$ Reproductive/late stage. Highlighted pathways (dashed boxes) include photosynthesis (PS), tricarboxylic acid cycle (TCA), pentose phosphate pathway (PPP), fatty acid degradation (FA), inositol phosphate metabolism (IPM), cysteine and methionine metabolism (CMM), nicotinamide metabolism (NM), starch and sucrose metabolism (SM), carbon fixation (CF), pyruvate metabolism (PM), photorespiration (PR), glyoxylate and dicarboxylate metabolism (GM), zeatin biosynthesis (ZB), biotin metabolism (BM), $\beta$-alanine metabolism (AM), taurine, and hypotaurine metabolism (TM). (C) KEGG-pathway enrichment of protein groups (Fisher's exact test; $p<0.05$ ) in vegetative and reproductive stages as compared to a customized reference of green plant species viz. Z. mays, G. max, A. thaliana, S. tuberosum, T. aestivum, N. tabacum, P. sativum, O. sativa, and H. vulgare

abscisic acid positively modulates the dormancy, whereas gibberellin enhances germination [39]. Moreover, underrepresentation of gibberellic acid dependent signaling pathways might be associated with picroside biosynthesis. A previous study reported that gibberellin downregulates the picroside biosynthesis as both follow the same biosynthetic routes i.e. mevalonate and non-mevalonate pathways [40]. Further, our initial observation suggests that $24.5 \%$ of $P$. kurroa proteome harbors putative RBPs that could be involved in plant development and adaptation to the alpine environment, however further experimental studies will be required.

It is well established that protein phosphorylation is an important PTMs that integrates external and internal stimuli, regulates carbon-nitrogen metabolism and redox signaling in plants [41]. As $P$. kurroa proteome was found to be extremely enriched for "response to abiotic stimulus" and "metabolic process", this provide us a clue that external abiotic stimuli might affect cellular and metabolic processes through protein phosphorylation in P. kurroa. However, ADP-ribosylation in the plant system has received less attention compared with the animal system, it plays a significant role in plant response to diverse biotic and abiotic stresses through implication in cellular processes like DNA repair [42]. Since, ADP-ribosylation can be catalyzed by ADP-ribosyl transferase in vitro and in vivo, the identification of 89 ribosylated peptides from $P$. kurroa provides information about the substrate 

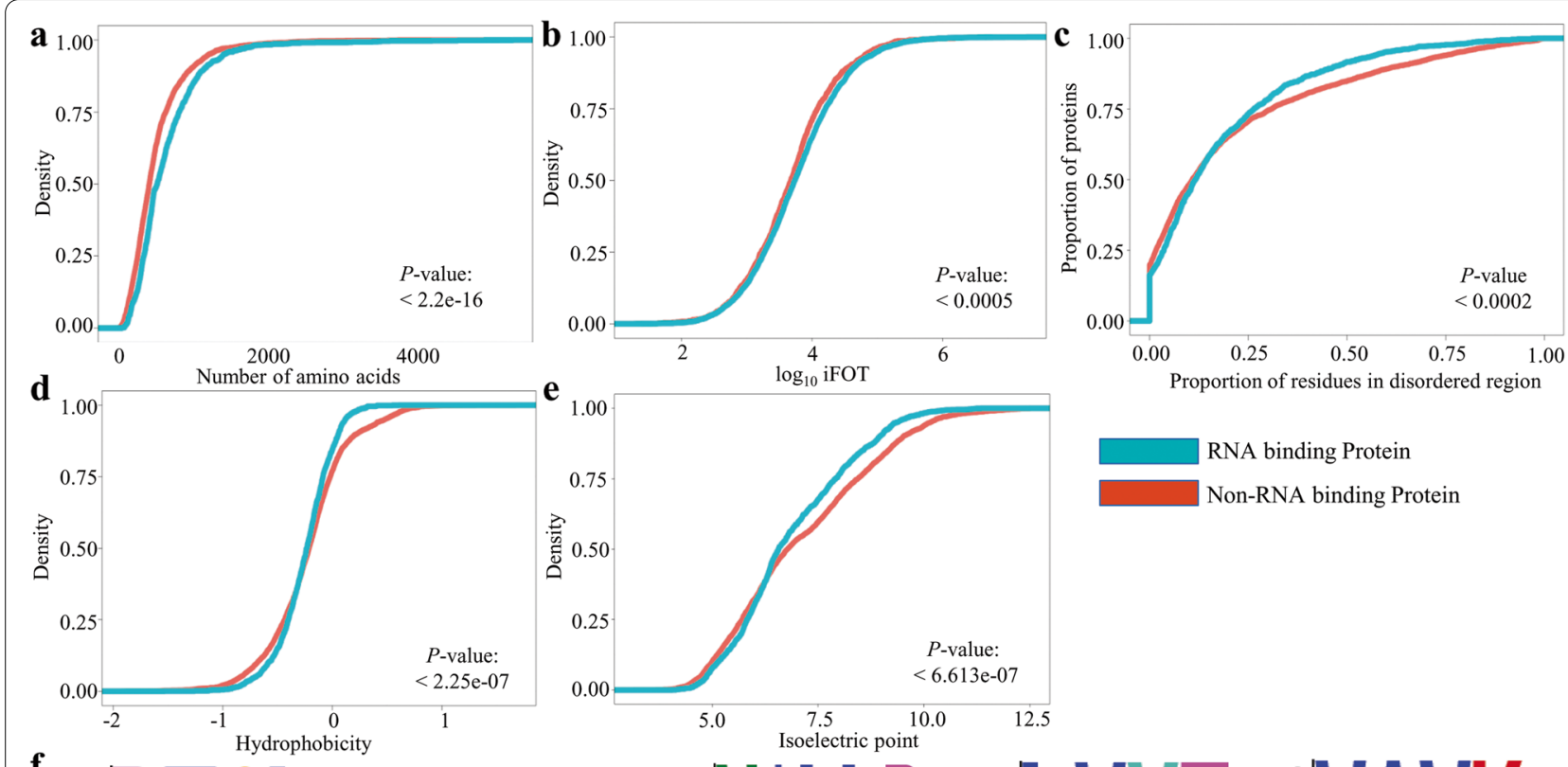

f
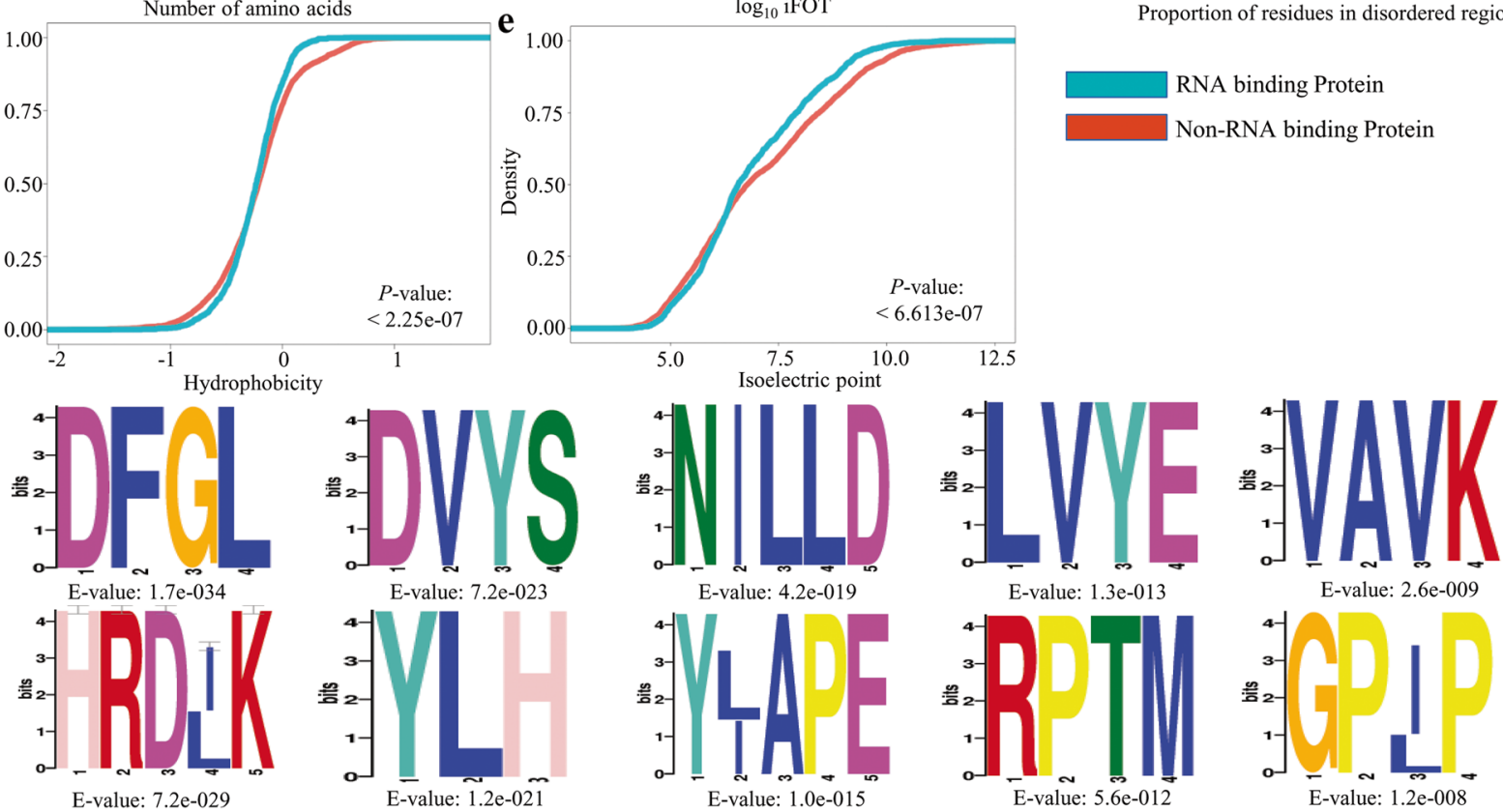

Fig. 6 Biophysical characteristics and sequence features of P. kurroa putative RBPs. A Density of protein length, B Density of $\log _{10}$ iFOT, C Proportion of residues in disorder region, $\mathbf{D}$ Hydrophobicity, and $\mathbf{E}$ Isoelectric point. Significant differences among putative RBPs and non-RBPs were analyzed using Kolmogorov-Smirnov test $(p<0.01)$. F The top ten most enriched amino acid motifs in $P$. kurroa putative RBPs analyzed by DREME software (E-value $<0.05)$

identification. Thus, we can hypothesize that PTMs particularly, phosphorylation and ADP-ribosylation beside others can play an important role in P. kurroa adaptation against environmental fluctuations prevailing at high altitude.

\section{Spatial profiling of $P$. kurroa organ proteomes reflect functional diversity}

To generate proteome map of P. kurroa, sub-proteomes of the four major organs were integrated. Moreover, individual proteome at vegetative and reproductive stages of development was incorporated for each organ, which represents organ sub-proteomes. We postulate that relatively higher abundance and specificity of proteins in an organ contribute significant organ specialized functions. For example, proteins associated with flower and seed development, fruit setting, flavonoid biosynthesis, embryogenesis, cell expansion, plant defense towards chilling and oxidative stress were found to be relatively abundant in inflorescence, which include cell wall invertase, methionine synthase, SCY1-like protein, transparent testa 12 , glutathione S-transferase tau 7, N-acetyltransferase HLS1-like, lysM domain receptor-like kinase 3, NAC transcription factor, pentatricopeptide repeatcontaining protein, exostosin family protein, F-box/LRRrepeat protein 10-like and AP2/B3-like transcriptional factor family protein [43-46]. Proteins linked with cell division, cell-cell communication, carbon fixation, system development, ubiquitination, signaling, protein folding, metabolism and stress response were found to be relatively more abundant in shoot. A few of these include ribulosebisphosphate carboxylase, carbonic anhydrase, long-chain acyl-CoA synthetase, WPP domain associated protein, MADS-box protein AGL19, stromal $70 \mathrm{kDa}$ heat shock related protein, glutathione transferase 23 , taxadiene 5-alpha hydroxylase, laccase precursor, LRR 
receptor-like serine/threonine protein kinase, two-component response regulator-like APRR2, RuBisCO large subunit-binding protein subunit alpha, F-box/kelchrepeat protein, chaperonin- 60 alpha and heat shock protein 70 .

Protein groups that comparatively abundant in root have a prominent biological role in root elongation, transport, root hair formation, differentiation, cell division, carbohydrate metabolism and defense response. The most abundant protein groups in root are alpha tubulin; significant impact on root development [47], ARF guanine-nucleotide exchange factor GNOM-like; involved in membrane trafficking and cytoskeleton dynamics to drive tip growth in root hairs [48] and others such as arabinogalactan 21 and transducin / WD-40 repeat family protein. Various channels/transporters like amino acid permease 4 (amino acid transport across membrane), potassium channel AKT6 (modulate root hair $\mathrm{K}^{+}$uptake), pleiotropic drug resistance protein 2-like (efflux antifungal or antibacterial metabolites), glutathione S-conjugate transporting ATPase (remove xenobiotics and endogenous toxicants out of cytosol) and $\mathrm{ABC}$ transporter $\mathrm{G}$ family member 11-like (metal transport and excretion of exudates) either enable the plant access for nutrients or impart defense response [49, 50]. Diverse signaling, cell cycle and defense/stress-responsive proteins abundant in root include peptidyl-prolyl cis-trans isomerase, receptor protein kinase, Phox (PX) domain, cucumisin-like, class III peroxidase, NAP1-like, MACPF domain-containing protein CAD1-like, HERK 1-like, phospholipid-transporting ATPase 9-like, LEA protein 2-like, SGT1-1, RPA $70 \mathrm{kDa}$ subunit, CDPK 33 and serine/threonine kinase that indirectly affect root growth and development [51]. This suggests that highaltitude alpine environment advances the proliferation of root system to gain access for soil water and nutrients, which would be useful in developing strategies for greater soil exploration capacities.

At ecosystem level, rhizome is associated with soil protection and prevents erosion [52]. It is well known that rhizome stores energy reserves (starch, proteins) and allocates nutrients for regrowth. The abundance pattern revealed proteins associated with secondary metabolic pathways (2-oxoglutarate-dependent dioxygenase AOP1.2-like and 4-coumarate-CoA ligase-like 1), energy metabolism (alpha subunit of ATPase, aconitate hydratase 3, ATP synthase CF1 beta subunit and stearoylACP desaturase), storage (concanavalin A-like lectin), defense/immunity (disease resistance RPP13-like, basic 7S globulin, MACPF domain-containing protein, thaumatin domain family, glucan endo-1,3-beta-glucosidase 12-like, O-glycosyl hydrolase family 17 and DUF231) and development in response to abiotic stimulus (chaperone protein dnaJ 20, glucomannan 4-beta-mannosyltransferase 9, ARM repeat superfamily and argininosuccinate lyase) were found to be relatively abundant in rhizome. Argininosuccinate lyase is essential for normal root elongation by arginine production besides ornithine accumulation through urea cycle under low $\mathrm{CO}_{2}$ availability [53]. Earlier, high expression of ARM family transcript has been reported specific in rhizome that promotes root growth [54]. Deep root elongation reduces the effects of environmental stresses and limits soil erosion. While, both rhizome and root perform mechanical and storage functions, the efficient growth and development of belowground system is not only associated with vegetative regeneration but also increases species survival under stressful environment. Therefore, each organ performs a specialized task in the life cycle of a plant, which is directly reflected in its proteome and deeply influenced by the environment.

\section{Metabolic pathway mapping and enrichment highlights functional differences between early and late developmental stages of $P$. kurroa}

Plant development is divided into vegetative (early) and reproductive (late) phases. Generally, the vegetative phase starts with pollination and ends with the commencement of flowering, whilst the reproductive phase begins with appearance of flowering organs and stops with pollination [55]. The enrichment of photosynthetic and Calvin cycle pathways in the vegetative phase will likely increase carbon availability that results increased plant growth. As plant grows from vegetative to generative stage, there is an increasing mass of reproductive organ that does not perform photosynthesis [56]. In that phase, plants carry temperature dependent respiration rate and thus, enhancement in glycolytic process and pyruvate metabolism were noticed in late stage to produce energy reservoirs. Photosynthesis is intricately associated with carbon fixation, however carbon assimilation process found to be relatively enriched in generative phase. Higher carbon fixation rate permit photosynthesis even in low $\mathrm{CO}_{2}$ to maintain resource use efficiency [57]. Although, RuBisCo catalyzes the carboxylation of ribulose-1,5-bisphosphate (RuBP) in photosynthesis, but also competes for oxygenation in photorespiration, thereby decreases photosynthetic efficiency. Photorespiration was found to be significantly enriched in the reproductive stage of $P$. kurroa, however less information is available about the orchestration of photorespiratory cycle during developmental phases. An earlier study reported that photorespiratory pathway is activated under fluctuating light conditions to benefit photosynthetic carbon fixation through regulation of electron flow [58]. In vegetative organs, lipid breakdown and phosphatidylinositol 
signaling might overcome the detrimental effects of free fatty acids and reactive oxygen species (ROS) that generate adaptive potential to adverse conditions [59]. Furthermore, enhanced cysteine biosynthesis might associate with glutathione pool in order to balance redox status of the cell. In addition, this could elevate the level of sulphur containing amino acids and improve the nutritional value of vegetative organs in P. kurroa. Reproductive development is more sensitive to abiotic stress as compared to vegetative phase, predominantly during fruit and seed setting [60]. Enrichment of metabolic pathways was found to be 1.6 folds on log scale in reproductive stage compared to vegetative. These metabolic pathways synthesized specialized compounds mostly during stationary growth phase and not implicated in reproduction or plant development. The pivotal role of sucrose and starch metabolism in plant development and stress response is well established as the interruption of sucrose signaling and metabolism caused significant reproductive failure in abiotic stress conditions [61]. On the whole, this reflects early stage is primarily associated with growth and development by utilizing less energy through alternative pathways, whereas the late stage harvests more energy in secondary metabolic pathways to synthesize diverse compounds of plant defense. Additionally, with reference to particular adaptive response, both early and late stage plants respond to environmental fluctuations through distinct processes.

\section{Accumulation of stress-responsive proteins may provide $P$. kurroa adaptation to stress combinations at high-altitude}

Our proteome map unravels many candidates responsive to abiotic and biotic stimuli, which are required for the synthesis of various regulatory/functionally defensive proteins and protective metabolites. These proteins accounted for $15.1 \%$ of the total identified proteome, which are of interest for $P$. kurroa, a potentially emerging model species for studying the adaptability to stress combinations prevailing at higher altitudes. Climate at highaltitudes are said to be harsh; specifically, low water and gases availability, fluctuating temperatures, high radiations, wind velocity and snow cover limit plant survival. Among functionally annotated stress-related proteins, response to stimulus (68.15\%), chemicals (28.26\%), stress (27.49\%), other organism (19.05\%), abiotic (15.08\%), biotic (10.86\%), light or radiation $(9.71 \%)$, ions (5.75\%), salt or osmotic stress (4.73\%) and cold temperature (4.47\%) were found to be over-represented in P. kurroa (Fig. 7A).

In response to alpine environment, $P$. kurroa could have some special stress-responsive proteins. Various classes of heat shock proteins (HSPs) including chloroplastic and mitochondrial localized, chaperonins and peptidyl-prolyl isomerase have been identified. These molecular chaperones facilitate protein folding, assist translocation and degradation of damaged ones under stress conditions. Additionally, heat shock factors were also observed that regulate the expression of these HSPs. To detoxify ROS and maintain redox homeostasis, the key enzymatic antioxidants like catalase, monodehydroascorbate reductase, dehydroascorbate reductase, glutathione peroxidase, glutathione reductase, glutathione S-transferase, 2-Cys peroxiredoxins and osmotin-like are evident in P. kurroa (Table S1). In brief, environmental stress is recognized by stress sensors that mediate signal transduction cascade to regulate gene expression or metabolic pathways imparting stress endurance to plants. Various stress signaling proteins (14-3-3, GF, calreticulin and calmodulin) and downstream components like kinases (casein, His, Ser/Thr, wall-associated, glycerol, receptor like, LysM domain, diacylglycerol, CLAVATA, farnesol, MAPK, CDPK and CIPK), phosphatases (Ser/Thr, inositol phosphate, phosphatidylinositol and lipid phosphatase), transcription factors (TFs) and channels/transporters were detected that play a crucial role in orchestration of stress responses in P. kurroa. The identified TFs such as zinc finger proteins, ERF107, ERF053, ERF118, AP2/ERF and B3 domain, WRKY, AIL5, ABI5, ONAC010, bHLH131, MYC2, NAC, bZIP, ERF1B, ERF027, RAP2-11, MYB3R3, MYB1R1 and AP2/EREBP among others were considered to be involved in the regulation of stress-related gene expression [62]. The ABA biosynthetic proteins like zeaxanthin epoxidase, aldehyde oxidase, 9-cis-epoxycarotenoid dioxygenase, molybdenum cofactor sulfurase and its downstream signaling signatures PYL, PP2C, ABFs along with chloroplastic receptor $\mathrm{CHLH}$ were caught attention. The identification of ABA-induced transporters/channels like ABCG, peptide/nitrate, two pore $\mathrm{K}^{+}$channel, guard cell outward rectifying $\mathrm{K}^{+}, \mathrm{Na}^{+} / \mathrm{H}^{+}$ and cation- $\mathrm{H}^{+}$exchanger shed light on ABA-mediated stress tolerance by regulating stomatal movements [63]. In addition to water regulation by aerial parts through stomata, belowground uptake is found to be enhanced by root growth through the involvement of vacuolar $\mathrm{H}^{+}$-pyrophosphatase that increases water retention and emphasizes organ-specific adaptation mechanisms. In continuation, diverse aquaporins were also detected that are key players of plant-water relations. Apart from this, a large number of $\mathrm{ABC}$ transporters have been identified including vacuolar, mitochondrial and multidrug resistance-associated proteins, which indicate the characteristics of heavy metals (HMs) tolerance that could be further explored for phytoremediation. In accordance, other observed transporters such as copper transport family, pleiotropic drug resistance protein, metal-nicotianamine YSL, heavy metal ATPase, Nramp family, MATE 


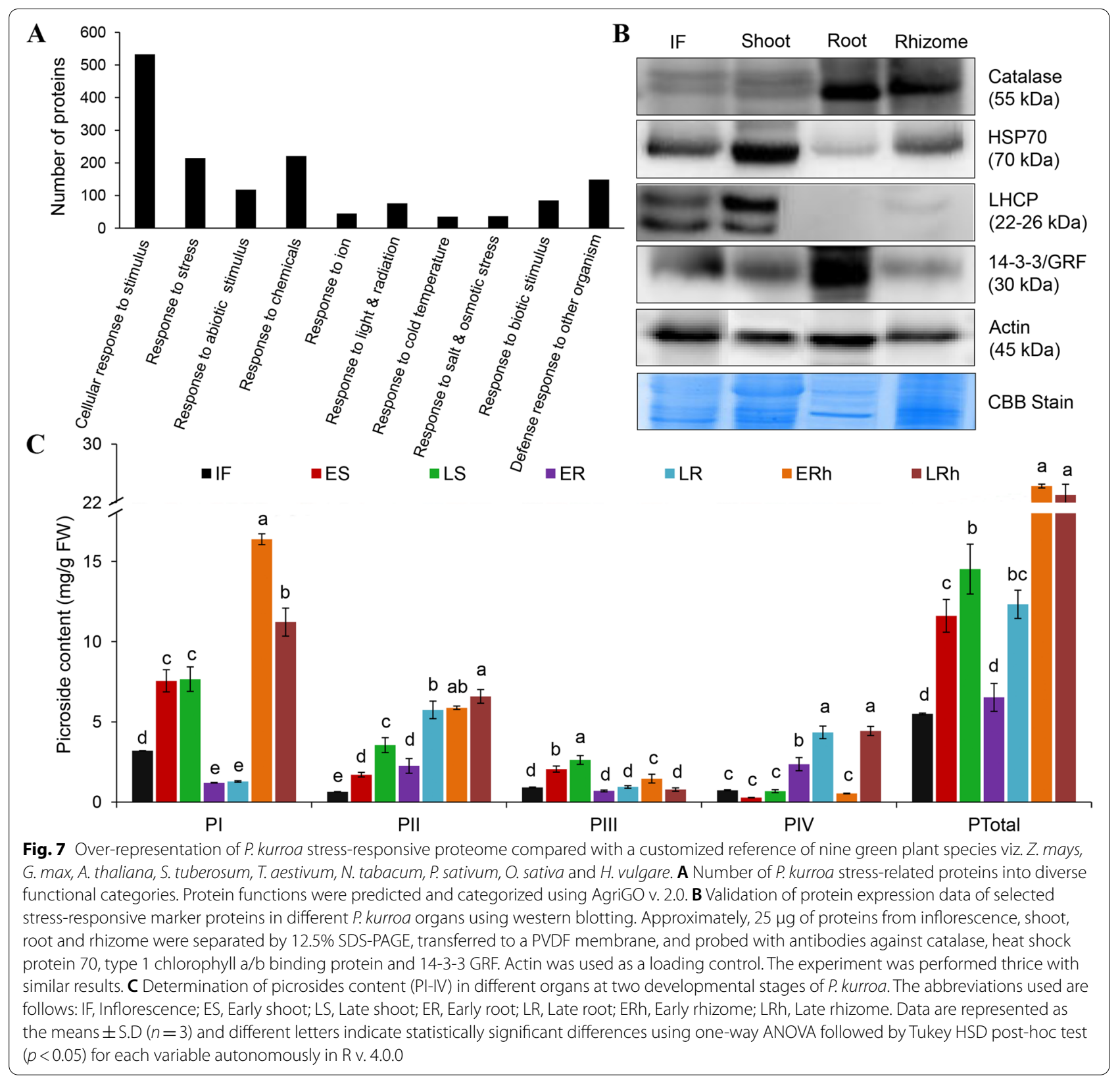

family, HM-associated isoprenylated protein and metal tolerance proteins are also considered to facilitate HMs tolerance [64].

Proteins involved in metabolic processes were found to be the largest group and of significant interest for understanding the adopted basal metabolism of alpine herbs. Stress metabolism, which results changes in bioenergetics is well documented in P. kurroa proteome. Besides the identification of an array of photosynthetic proteins, photorespiratory enzymes were more evident (Table S1), which is considered as a defense line at molecular level against photooxidative damage. In addition, secondary metabolic enzymes were well observed in $P$. kurroa that lead to the formation of diverse secondary metabolites (SMs) having protective functions from stresses such as anthocyanins, flavonoids/phenolics, glucosinolates, volatiles, lignins, oxylipins and others. Moreover, several protective proteins were also enriched such as pathogenesis-related, LEA family, stress-related, PLAT-plant stress domain, thioredoxin, lipoxygenase and lactoylglutathione lyase that enable $P$. kurroa to resist several abiotic and biotic stress combinations at high-altitudes. Further, western blotting was performed to validate the expression of stress-responsive proteins in different 
organs (Fig. 7B; Fig. S1). Subsequently, four stress-related proteins, namely heat shock protein 70 (HSP70), catalase, LHCP and 14-3-3 were selected for expression analysis. The western blotting data was in agreement with our proteomic results and discrepancies were observed among organs. HSP70 was universally expressed in all four organs with more abundance in shoot. Catalase was highly expressed in root and rhizome, whereas 14-3-3 abundance detected more in root. Shoot showed highest abundance for LHCP followed by inflorescence, which was not detected in root. Therefore, we conclude that stress-responsive proteins provide adaptive significance to $P$. kurroa towards harsh environment and might be potential targets for crop improvement under adverse conditions (Fig. 8).

\section{Proteins associated with secondary metabolism may provide medicinal significance to $P$. kurroa}

P. kurroa is enriched with many SMs in an organ-specific manner having diverse biological effects [7]. These SMs have thought to be key drivers of pharmacological actions, which provide the foundation for its use in the traditional and modern medicine system. Being a medicinal herb, $P$. kurroa proteome was found to be functionally enriched for small molecule metabolic process (289 proteins, $p=1.3 \mathrm{E}-23$ ), generation of precursor metabolites (81 proteins, $p=3.5 \mathrm{E}-12$ ), secondary metabolic process (35 proteins, $p=1.4 \mathrm{E}-08$ ), secondary metabolite biosynthetic process (24 proteins, $p=7.9 \mathrm{E}-07$ ), small molecule biosynthetic process (83 proteins, $p=3.7 \mathrm{E}-06$ ) and terpenoid metabolic process (17 proteins, $p=5.6 \mathrm{E}$ 06). This revealed that proteins associated with SMs, especially phenolics, flavonoids, alkaloids, terpenoids, iridoids, glucosinolates and plant hormones were mostly identified in rhizome compared to other organs. At organ level, proteins linked with phenolics were predominant in rhizome, while those associated with terpenoids were greatest in the shoot. Terpenoid biosynthesis is an important function in P. kurroa, which is found to be operated mostly in the shoot by the involvement of both mevalonate and non-mevalonate pathways. Apart from this, several identified proteins were mapped on the unique KEGG metabolism revealing organ-specificity at pathway level such as proteins involved in $\alpha$-linoleic acid metabolism, lipopolysaccharide biosynthesis, $\beta$-alanine metabolism, pantothenate biosynthesis, taurine and hypotaurine metabolism and isoquinoline alkaloid biosynthesis were exclusively identified in the rhizome. While, proteins play a key role in zeatin biosynthesis and methane metabolism were observed in root and inflorescence, respectively. However, proteins related to photosynthesis, sesquiterpenoid/triterpenoid biosynthesis and biotin metabolism were detected uniquely in the shoot.

Of particular interest, $P$. kurroa is known for its specialized metabolites i.e. picrosides. The picroside biosynthesis is associated with mevalonate, non-mevalonate, iridoid and shikimate/phenylpropanoid pathways. Two proteins of plastidic non-mevalonate pathway like 1-deoxy-D-xylulose-5-phosphate synthase and 4-diphosphocytidyl-2-C-methyl-D-erythritol kinase were identified only in the shoot, which might synthesize picrosides. The cytosolic mevalonate pathway proteins such as acetyl-CoA acetyltransferase, 3-hydroxy-3-methylglutaryl-coenzyme A reductase 2 and acetoacetyl-CoA thiolase 1 were observed in rhizome and shoot, respectively. In addition, proteins belong to shikimate/pheylpropanoid pathways such as shikimate O-hydroxycinnamoyltransferase, phospho-2-dehydro-3-deoxyheptonate aldolase 2, chorismate mutase, arogenate dehydratase were detected in shoot and rhizome. Furthermore, phenylalanine

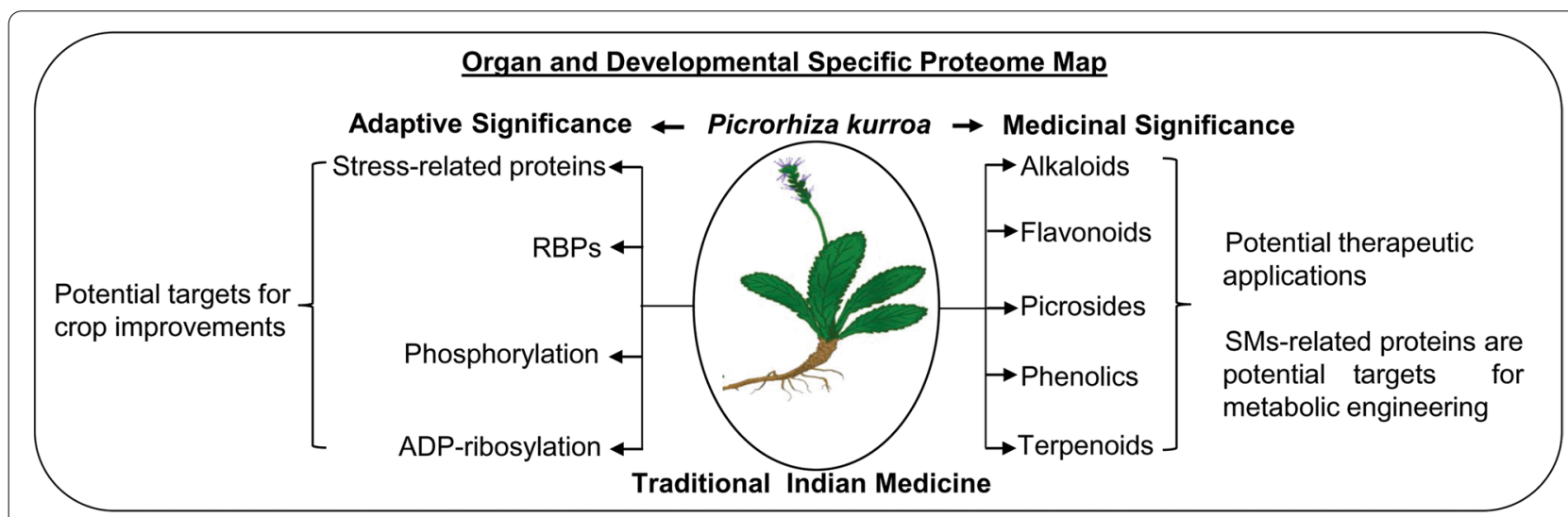

Fig. 8 Organ and development dissected proteome map of $P$. kurroa reveals medicinal and adaptive significance. A quantitative analysis of proteins associated with stress-response and synthesis of secondary metabolites are potential targets for crop improvement and metabolic engineering, respectively 
ammonia-lyase, a key enzyme involved in secondary metabolism and phenolics accumulation was found in the rhizome, which might produce a variety of SMs. While, the main enzyme of iridoid pathway, heterodimeric geranylgeranyl pyrophosphate synthase was identified in all organs, suggesting all $P$. kurroa organs contributed in picroside biosynthesis. Thus, to gain more insights into metabolic differences among $P$. kurroa organs at different developmental phases, the marker compounds viz. picrosides (I-IV) were quantified using UHPLC-PDA system (Fig. 7C; Fig. S2). Among four picrosides, PI content was found to be highest followed by PII. Additionally, a significant variation was found to be associated with both organ and developmental stages. The highest content of PI was observed in rhizome followed by shoot, inflorescence and root. The maximum level of PII was observed in rhizome, which slightly reduced in root followed by shoot and inflorescence. However, the quantity of PIII and PIV was less, while the greatest amount was detected in shoot and root, respectively. The picroside content also varies significantly with the stage of development. In rhizome, PI and PIII decreased, while PII and PIV increased significantly at the late stage compared to an early phase. Also, a significant enhancement was noticed for PII and PIV in root and PII and PIII in shoot at the late stage of development. As a result, total picroside content elevated significantly at the generative phase in root and shoot, while no significant change was observed in rhizome. Overall, total picroside content was highest in rhizome followed by shoot compared to other organs, which is in line with our proteomic data. These secondary metabolisms related proteins would be potential targets for enhanced specialized metabolites production through metabolic engineering (Fig. 8).

\section{Conclusions}

This study is navigated towards the systematic investigation of whole proteome in high-altitude medicinal herb, P. kurroa. The use of 1D-GE and nanoLC-MS/MS enabled us to achieve the deep proteome coverage with 5186 protein accessions enriched for 359 GO terms, when compared with green plants so far. The quantitative analyses of sub-proteomes across four major organs with regard to biological functions and protein abundance provide a comprehensive map of organ-specific and shared functions. Also, pathway-centric functional mapping at key developmental stages highlights the distinct adaptive processes to alpine environmental perturbations. Deep analysis of stress-responsive biological functions provides several candidate proteins involved in multiple stress tolerance that could be potential targets for crop improvement. Additionally, proteomic analysis led to the detection of several proteins linked with secondary metabolism for the synthesis of picrosides, flavonoids, alkaloids, phenolics and terpenoids that could offer novel opportunities of metabolic engineering for enhanced SMs production. P. kurroa proteome profiling will enrich the proteome database and presents a good groundwork for further studies with an ultimate goal of complete understanding of complex molecular networks for specialized metabolites production and combinatorial stress tolerance.

\section{Methods}

\section{Plant sampling}

P. kurroa was collected from Rohtang pass in Pir Panjal range of Western Himalaya, India $\left(32^{\circ} 23^{\prime} 30^{\prime \prime} \mathrm{N}\right.$, $77^{\circ} 15^{\prime} 24^{\prime \prime E}$; 3400 masl) in July 2017 and 2019 during an extended noon period. India's Biological Diversity Act 2002 permits bonafide Indians to access biological resources for scientific research [65]. The voucher specimen has been deposited in an internationally recognized herbarium of CSIR-IHBT, Palampur with acronym 'PLP'. The identification of plant specimen was performed by taxonomist of our institute and complete information of voucher specimen like scientific name, Picrorhiza kurroa Royle ex Benth.; common name, Kadu; family, Plantaginaceae; place of plant collection, Rohtang Pass was recorded under specimen no. 15384 as described previously [7]. Four different organs viz. shoot, root, rhizome and inflorescence at two developmental stages i.e. vegetative (before flowering) and reproductive (after flowering) were collected, instantly frozen in liquid nitrogen and stored at $-80{ }^{\circ} \mathrm{C}$ until use. To construct $P$. kurroa proteome map, at least eight independent plants of each growth stage (vegetative and reproductive) were used for dissecting morphologically differentiated four plant organs.

\section{Protein extraction and determination}

Protein extraction from different organs (shoot, root, rhizome and inflorescence) at key developmental stages was performed as explained by Kumar et al. [66]. Approximately, $2 \mathrm{~g}$ of each frozen sample was ground to fine powder in liquid nitrogen and transferred into prechilled centrifuge tube having $10 \mathrm{ml}$ of homogenization buffer [Sucrose (40\%), $1 \mathrm{mM}$ EDTA (pH 7.5), $50 \mathrm{mM}$ HEPES-KOH (pH 7.5), Triton X-100 (1\%), 1 mM PMSF, $\beta$-mercaptoethanol (1\%) and $10 \mathrm{mM}$ ascorbic acid]. The homogenate was vortexed and stirred with tris-equilibrated phenol ( $\mathrm{pH} 8.0)$ on rocker for $30 \mathrm{~min}$ prior to centrifugation at $5000 \mathrm{~g}$ for $15 \mathrm{~min}$. The phenol phase was separated and precipitated overnight with $0.1 \mathrm{M}$ ammonium acetate in methanol. Through centrifugation at $10,000 \mathrm{~g}$ for $15 \mathrm{~min}$, protein pellets were recovered, cleansed with $80 \%$ acetone twice, air dried and solvated 
in rehydration buffer [8 $\mathrm{M}$ urea, CHAPS (2\%) and $2 \mathrm{M}$ thiourea]. All steps were conducted on ice or at $4{ }^{\circ} \mathrm{C}$. Protein concentration was determined by Bradford method with BSA as a calibration standard [67].

\section{SDS-PAGE and nanoLC-MS/MS}

Approximately, $25 \mu \mathrm{g}$ of each protein sample were dissolved in sample buffer [0.25 M Tris-Cl (pH 6.8), glycerol (50\%), SDS (10\%), $0.5 \mathrm{M}$ dithiothreitol (DTT), bromophenol blue $(0.25 \%)]$ and resolved by SDS-PAGE on $12.5 \%$ polyacrylamide gels (Biorad, India). Subsequently, fractionated proteins were visualized with $\mathrm{CBB}$ and each sample lane was excised into eight fractions. In-gel digestion was performed according to a standard protocol [68]. The excised gel pieces were destained with $100 \mathrm{mM}$ $\mathrm{NH}_{4} \mathrm{HCO}_{3}$ and acetonitrile (ACN) followed by washing with water thrice. The gel pieces were reduced with $10 \mathrm{mM}$ DTT in $100 \mathrm{mM} \mathrm{NH}_{4} \mathrm{HCO}_{3}$ at $60^{\circ} \mathrm{C}$ for $45 \mathrm{~min}$, alkylated with $50 \mathrm{mM}$ iodoacetamide (IAA) in $100 \mathrm{mM}$ $\mathrm{NH}_{4} \mathrm{HCO}_{3}$ at $\mathrm{RT}$ in dark for $20 \mathrm{~min}$ followed by washing with $100 \mathrm{mM} \mathrm{NH} \mathrm{HCO}_{3}$. Trypsin digestion was carried out with $10 \mathrm{ng} / \mu \mathrm{L}$ trypsin (Promega, India) in $50 \mathrm{mM}$ $\mathrm{NH}_{4} \mathrm{HCO}_{3}$ at $37^{\circ} \mathrm{C}$ for $12-16 \mathrm{~h}$. Peptides were extracted with $0.1 \%$ trifluoroacetic acid in $\mathrm{H}_{2} \mathrm{O}: \mathrm{ACN}$ (50:50) and lyophilized (Labconco, USA).

For nanoLC-MS/MS, tryptic peptides were redissolved in $0.1 \% \mathrm{HCOOH} / \mathrm{ACN}$ and loaded on BEH C18 column $(1.7 \mu \mathrm{m}, 75 \mu \mathrm{m} \times 200 \mathrm{~mm}$; Waters, India) equilibrated for $3 \mathrm{~min}$ with $0.1 \% \mathrm{HCOOH}$ having a flow rate of $5 \mu \mathrm{L} / \mathrm{min}$. NanoLC-MS/MS was performed with HPLC chip coupled to a 6560 Q-TOF (Agilent Technologies, USA). A linear gradient of $75 \mathrm{~min}$ was employed for peptide separation by a series of 1200 nano pump (Agilent Technologies, Santa Clara, USA) through two solvent system comprises A; $0.1 \%$ $\mathrm{HCOOH}$ in water and $\mathrm{B} ; 0.1 \% \mathrm{HCOOH}$ in acetonitrile. The gradient program starts with $5-8 \mathrm{~min}, 3-15 \% \mathrm{~B} ; 8-50 \mathrm{~min}$, $15-45 \%$ B; $50-55 \mathrm{~min}, 45-90 \%$ B, hold for $5 \mathrm{~min}$ and finally resume within $1 \mathrm{~min}$. The subsequent parameters were placed in the acquisition mode: MS scan range $(\mathrm{m} / \mathrm{z})$ and rate (spectra/sec), 50-1700 and 3; MS/MS scan range (m/z) and rate (spectra/sec), 50-1700 and 1; collision energy, a slope of $3.6 \mathrm{~V}$ and an offset of $4.8 \mathrm{~V}$. While, the following criteria were specified for precursor selection: threshold (Abs), 5000; max. precursor per cycle, 3; threshold (Rel. \%), 0.010; active exclusion excluded after 2 spectra and released after $0.5 \mathrm{~min}$; target (counts/spectrum), 25,000; preferred charge state, $2,3,>3$. The instrument parameters were set at positive ionization mode, Gas Temp $\left({ }^{\circ} \mathrm{C}\right), 325$; Gas Flow (l/min) 6; capillary voltage, $2000 \mathrm{~V}$ and skimmer, $65 \mathrm{~V}$.

\section{Protein identification and quantification}

Raw data (.d files) were analyzed using Spectrum Mill Proteomics Workbench software (Agilent Technologies,
USA) that extracts spectra, MS/MS searches for spectra and validates peptides or proteins in the database. The default extraction parameters used were: $\mathrm{MH}^{+}$precursor range (Da), 600-4000; scan time range ( $\mathrm{min}), 0-300$; time and mass window for merging scans by spectral similarity and RT and $m / z, \pm 60 \mathrm{~s}$ and $\pm 1.4 \mathrm{~m} / z$. MS/MS search criteria were modified as: database, NCBInr; species, plants; fixed modification, carbamidomethylation (C); match filtering, disabled; mass type, monoisotopic; min. matched peak intensity (\%), 50; max. ambiguous precursor charge, 3; precursor mass tolerance (ppm), 20; product mass tolerance (ppm), 50; search mode, identity with enabled calculate reversed database scores. MS/MS auto validation was performed on peptide mode with FDR $<1 \%$. The results were shown in "protein/peptide summary report" after filtered by peptide score threshold $\geq 3.0$ and percentage scored peak intensity (\% SPI) threshold $\geq 60 \%$. An intensity-based absolute quantification algorithm (iBAQ) was used to calculate the protein abundance [69]. The protein intensity was first calculated by Spectrum Mill software as a total of the entire identified peptide intensities. Then, protein intensity was divided by the theoretically observable peptides number (evaluated by in silico protein digestion using MS digest tool and all tryptic peptides length between 6-30 amino acids were considered). The resulted intensity was shown as "absolute iBAQ intensity" (iBAQ). For sample to sample comparison and data normalization, the "relative iBAQ intensity/fraction of total iBAQ" (FOT-iBAQ) of each protein was assessed by dividing the absolute $\mathrm{iBAQ}$ intensity by a total of absolute iBAQ intensities of the entire proteins in the sample. Finally, FOT-iBAQ value was multiplied by $10^{5}$ to get "intensity-based fraction of total" (iFOT) value for simple visualization of low abundant proteins [70]. The estimated iFOT represented the abundance of protein in each organ.

\section{Bioinformatics and statistical analysis}

A circular proteome map of $P$. kurroa was generated for four morphologically differentiated organs using Circos (v.0.69-9) [71]. The assignment of protein functions was based on (GO) categories of nine plant species (identified for $P$. kurroa total proteome) covering Z. mays, G. max, $A$. thaliana, S. tuberosum, N. tabacum, T. aestivum, P. sativum, O. sativa and H. vulgare. Enrichment analysis of GO categories was performed in AgriGO (v.2.0) using Fisher's exact test $(p<0.05)$ [72]. Over and under-representation of GO categories was computed for total proteome (5186 identified proteins) as compared to a customized reference proteome, which includes aforementioned nine plant species. The proteome of each organ was also compared to total proteome for assessing organ-specific enriched GO terms $(p<0.05)$. PCA and correlation study were 
assessed in R (v.4.0.0) based upon the protein abundance. Hierarchical clustering analysis was also implemented in $\mathrm{R}$ (v.4.0.0) for those proteins present in at least two organs and an optimum number of clusters were obtained based upon Silhouette index (0.66). KEGG pathway mapping software iPATH (v.3.0) was used to visualize the differences between developmental stages at metabolic pathway level [73]. Also, pathway enrichment was performed for protein groups of both stages against aforementioned plant species using ShinyGO (v.0.61) [74]. ModPred tool was used to identify peptides containing PTMs with high confidence (false positive rate of 0.01) [75]. Next, domain information was fetched for all identified proteins from Pfam database [76]. Notable biophysical properties of RBPs such as protein length, isoelectric point, hydrophobicity, disordered regions were assessed using ProtParam (https://web.expasy.org/protparam), isoelectric point calculator, protein GRAVY and IUPred2A, respectively [7779]. The comparative analysis of biophysical properties between RBPs and non-RBPs was performed using Kolmogorov-Smirnov test at $1 \%$ level of significance. Also, a motif enrichment analysis was performed for 1272 putative RBPs using DREME software (v.5.1.1) with a threshold E-value of 0.05 [80].

\section{Western blotting}

To validate the proteins expression, the total extracted proteins from four different organs viz. inflorescence, shoot, root and rhizome were used for immunoblotting. Briefly, $25 \mu \mathrm{g}$ of protein were resolved onto $12.5 \%$ SDSPAGE and transferred onto $0.45 \mu \mathrm{m}$ PVDF membrane

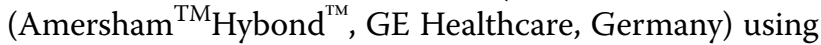
a wet transblot system. Afterwards, membranes were blocked with $2.5 \%$ non-fat dry milk in TBST $(10 \mathrm{mM}$ Tris-HCl, pH 7.5; $15 \mathrm{mM} \mathrm{NaCl} ; 0.05 \%$ tween 20 ) at $4{ }^{\circ} \mathrm{C}$ overnight, washed thrice and probed with rabbit polyclonal antibodies raised against catalase (Agrisera AS09 501), HSP70 (Agrisera AS08 371), LHCA1 (PS1 chlorophyll a/b-binding protein, Agrisera AS01 005), 14-3-3 GRF (Agrisera AS12 2119) and actin (Agrisera AS13 2640) followed by incubation with horseradish peroxidase (HRP)-conjugated goat anti-rabbit secondary antibody (Agrisera, AS09 602) for $1 \mathrm{~h}$ at RT. Blots were developed using ECL ${ }^{\mathrm{TM}}$ Prime detection reagents (GE Healthcare, Germany) and visualized under an Azure c300 Chemiluminescent Imaging Biosystem (California, USA).

\section{Determination of picrosides content in P. kurroa}

Approximately, $100 \mathrm{mg}$ fresh weight sample was extracted in $70 \%$ methanol and quantitative analysis of picrosides was performed using UHPLC-PDA system (Agilent, Santa Clara, USA) as described previously [7].
Abbreviations

ACN: Acetonitrile; CBB: Coomassie brilliant blue; DTT: Dithiothreitol; FDR: False discovery rate; GO: Gene ontology; IAA: lodoacetamide; iBAQ: Intensity-based absolute quantification; iFOT: Intensity-based fraction of total; LC: Liquid chromatography; MS: Mass spectrometry; 1D-GE: One-dimensional gel electrophoresis; PI: Picroside I; PII: Picroside II; PIII: Picroside III; PIV: Picroside IV; PCA: Principal component analysis; PTMs: Post-translational modifications; Q-TOF: Quadrupole-time of flight; RBPs: RNA binding proteins; SDS-PAGE: Sodium dodecyl sulphate-polyacrylamide gel electrophoresis; SMs: Secondary metabolites; SPI: Scored peak intensity; 2D-GE: Two-dimensional gel electrophoresis.

\section{Supplementary Information}

The online version contains supplementary material available at https://doi. org/10.1186/s12870-021-03394-8.

Additional file 1: Table S1. List of proteins identified through 1D-GEnanoLC-MS/MS in different organ and developmental dissected samples of P. kurroa.

Additional file 2: Table S2. List of protein accessions with their calculated relative abundance (iFOT) in each of the organ proteomes.

Additional file 3: Table S3. Gene ontology enrichment analyses of whole proteome and organ proteomes using AgriGO (Fisher's exact test and Bonferroni adjustment, $p<0.05)$. GO categories type: $C$, Cellular component; F, Molecular function; $P$, Biological process.

Additional file 4: Table S4. Five protein clusters were generated by hierarchical clustering analysis (silhouette index $>0.66$ ) comprised of protein accessions, their description along with calculated Z-scores in each of the organ.

Additional file 5: Table S5. Proteome-wide analysis of protein domains and screened RNA-binding domains (RBDs) using Pfam database. A list of P. kurroa putative RNA-binding proteins (RBPs) and their motif enrichment using DREME software (E-value $<0.05)$.

Additional file 6: Table S6. List of different post-translational modifications observed at peptide level using ModPred. Also, detailed list of ADP-ribosylated and phosphorylated peptides and their protein identifiers along with modified residues.

Additional file 7: Fig. S1. Western blots and gel images in raw form for all the organ samples. The abbreviations used are as follows: IF, Inflorescence; S, Shoot; R, Root; Rh, Rhizome; M, Marker lane (10-250 kDa).

Additional file 8: Fig. S2. UHPLC chromatograms of picrosides extracted in $70 \%$ methanol at $270 \mathrm{~nm}$. The abbreviations used are: IF, Inflorescence; ES, Early shoot; LS, Late shoot; ER, Early root; LR, Late root; ERh, Early rhizome; LRh, Late rhizome; PI, Picroside I; PII, Picroside II; PIII, Picroside III; PIV, Picroside IV.

\section{Acknowledgements}

We thank to Director of CSIR-IHBT for providing the research infrastructure; Zeeshan Z. Banday for critical reading and correction of manuscript. MK acknowledges UGC for providing research fellowship in Ph.D.

\section{Authors' contributions}

RK conceived and designed the experiment. RK, MK, RJ and AP conducted experiments. MK, UKP and RS performed bioinformatics and statistical analysis. RK and MK analyzed the data and wrote the manuscript. All authors edited and approved the manuscript.

\section{Funding}

This work was supported by Department of Science and Technology, SERBECR (Grant, ECR/2017/000771) and CSIR funded project (MLP-0201). The funder has no any role in designing of the experiment, wet lab experiments, data analysis, and writing of the manuscript.

\section{Availability of data and materials}

All the data are presented in this manuscript and in the supporting files are available via ProteomeXchange with identifier PXD030263. 


\section{Declarations}

\section{Ethics approval and consent to participate}

This study uses plant material and has been not involved any transgenic plant. All the plant materials were collected from natural habitat.

\section{Consent for publication}

Not applicable

\section{Competing interests}

Authors have no conflict of interest to declare.

\begin{abstract}
Author details
${ }^{1}$ Biotechnology Division, CSIR-Institute of Himalayan Bioresource Technology (IHBT), Palampur 176061, HP, India. ${ }^{2}$ Academy of Scientific and Innovative Research (AcSIR), Ghaziabad 201002, India. ${ }^{3}$ Studio of Computational Biology \& Bioinformatics (Biotech Division), The Himalayan Centre for High-throughput Computational Biology (HiCHiCoB, A BIC Supported by DBT, India), CSIR-IHBT, Palampur, HP, 176061, India. ${ }^{4}$ Present address: ICAR-Indian Agricultural Statistics Research Institute, Library Avenue, Pusa, New Delhi, Delhi 110012, India.
\end{abstract}

Received: 24 July 2021 Accepted: 12 December 2021

Published online: 22 December 2021

\section{References}

1. Debnath P, Rathore S, Walia S, Kumar M, Devi R, Kumar R. Picrorhiza kurroa: A promising traditional therapeutic herb from higher altitude of western himalayas. J Herb Med. 2020;23:100358.

2. Soni D, Grover A. "Picrosides" from Picrorhiza kurroa as potential anticarcinogenic agents. Biomed Pharmacother. 2019;109:1680-7.

3. Kumar R, Kumari M. Adaptive mechanisms of medicinal plants along altitude gradient: contribution of proteomics. Biol Plant. 2018;62(4):630-40.

4. Sah JN, Varshney VK. Chemical constituents of Picrorhiza genus. Am J Essent Oil. 2013;1(2):22-37.

5. Mehra TS, Chand R, Sharma Y. Reproductive biology of Picrorhiza kurroa-a critically endangered high value temperate medicinal plant. Open Access J Med Aromat Plants. 2010;1(2):40-3.

6. Kumar V, Bansal A, Chauhan RS. Modular design of Picroside-II biosynthesis deciphered through NGS transcriptomes and metabolic intermediates analysis in naturally variant chemotypes of a medicinal herb, Picrorhiza kurroa. Front Plant Sci. 2017;8:564.

7. Kumari M, Joshi R, Kumar R. Metabolic signatures provide novel insights to Picrorhiza kurroa adaptation along the altitude in himalayan region. Metabolomics. 2020;16(7):1-17.

8. Thani PR, Sharma YP, Kandel P. Phytochemical studies on Indian market samples of drug "Kutki" Picrorhiza kurroa Royle ex Benth. Res J Agric Forestry Sci. 2018;6(2):1-5.

9. Liska AJ, Shevchenko A. Expanding the organismal scope of proteomics: Cross-species protein identification by mass spectrometry and its implications. Proteomics. 2003:3(1):19-28.

10. Carpentier SC, Witters E, Laukens K, Van Onckelen H, Swennen R, Panis B. Banana (Musa spp.) as a model to study the meristem proteome: acclimation to osmotic stress. Proteomics. 2007;7(1):92-105.

11. Sharmin SA, Alam I, Rahman MA, Kim KH, Kim YG, Lee BH. Mapping the leaf proteome of Miscanthus sinensis and its application to the identification of heat-responsive proteins. Planta. 2013;238(3):459-74.

12. Schiltz S, Gallardo K, Huart M, Negroni L, Sommerer N, Burstin J. Proteome reference maps of vegetative tissues in pea. An investigation of nitrogen mobilization from leaves during seed filling. Plant Physiol. 2004;135(4):2241-60.

13. Uvackova L, Ondruskova E, Danchenko M, Skultety L, Miernyk JA, Hrubík P, Hajduch M. Establishing a leaf proteome reference map for Ginkgo biloba provides insight into potential ethnobotanical uses. J Agric Food Chem. 2014;62(47):11547-56.

14. Donnelly BE, Madden RD, Ayoubi P, Porter DR, Dillwith JW. The wheat (Triticum aestivum L.) leaf proteome. Proteomics. 2005;5(6):1624-33.

15. Katam R, Basha SM, Suravajhala P, Pechan T. Analysis of peanut leaf proteome J Proteome Res. 2010:9(5):2236-54.
16. Valero Galvan J, Valledor L, González Fernandez R, Navarro Cerrillo RM, Jorrin-Novo JV. Proteomic analysis of holm oak (Quercus ilex Subsp. Ballota [Desf.] Samp.) pollen. J Proteomics. 2012;75(9):2736-44.

17. Valledor L, Castillejo MA, Lenz C, Rodríguez R, Canal MJ, Jorrin J. Proteomic analysis of Pinus radiata needles: 2-DE map and protein identification by LC/MS/MS and substitution-tolerant database searching. J Proteome Res. 2008:7(7):2616-31.

18. Quiala E, Cañal MJ, Rodríguez R, Yagüe N, Chávez M, Barbón R, Valledor L. Proteomic profiling of Tectona grandis $\mathrm{L}$. leaf. Proteomics. 2012;12(7):1039-44

19. Giavalisco P, Nordhoff E, Kreitler T, Klöppel K, Lehrach H, Klose J, Gobom J. Proteome analysis of Arabidopsis thaliana by two-dimensional gel electrophoresis and matrix-assisted laser desorption/ionisation-time of flight mass spectrometry. Proteomics. 2005:5(7):1902-13.

20. Tanaka N, Fujita M, Handa H, Murayama S, Uemura M, Kawamura Y, Mitsui T, Mikami S, Tozawa Y, Yoshinaga T. Proteomics of the rice cell: systematic identification of the protein populations in subcellular compartments. Mol Genet genomics. 2004:271(5):566-76.

21. Brechenmacher L, Lee J, Sachdev S, Song Z, Nguyen THN, Joshi T, Oehrle $\mathrm{N}$, Libault M, Mooney B, Xu D. Establishment of a protein reference map for soybean root hair cells. Plant Physiol. 2009;149(2):670-82.

22. Xu J, Pascual L, Aurand R, Bouchet J, Valot B, Zivy M, Causse M, Faurobert M. An extensive proteome map of tomato (Solanum lycopersicum) fruit pericarp. Proteomics. 2013:13(20):3059-63.

23. Mathesius U, Keijzers G, Natera SHA, Weinman JJ, Djordjevic MA, Rolfe BG. Establishment of a root proteome reference map for the model legume Medicago truncatula using the expressed sequence tag database for peptide mass fingerprinting. Proteomics. 2001;1(11):1424-40.

24. Guo B, Chen Y, Li C, Wang T, Wang R, Wang B, Hu S, Du X, Xing H, Song $X$. Maize (Zea mays L.) seedling leaf nuclear proteome and differentially expressed proteins between a hybrid and its parental lines. Proteomics. 2014;14(9):1071-87.

25. Méchin V, Balliau T, Château-Joubert S, Davanture M, Langella O, Négroni L, Prioul JL, Thévenot C, Zivy M, Damerval C. A two-dimensional proteome map of maize endosperm. Phytochemistry. 2004;65(11):1609-18.

26. Mergner J, Frejno $M$, List M, Papacek M, Chen $X$, Chaudhary A, Samaras $P$, Richter S, Shikata H, Messerer M. Mass-spectrometry-based draft of the Arabidopsis proteome. Nature. 2020:579(7799):409-14.

27. Duncan O, Trösch J, Fenske R, Taylor NL, Millar AH. Resource: Mapping the Triticum aestivum proteome. Plant J. 2017;89(3):601-16.

28. Carpentier SC, Panis B, Vertommen A, Swennen R, Sergeant K, Renaut J, Laukens K, Witters E, Samyn B, Devreese B. Proteome analysis of nonmodel plants: a challenging but powerful approach. Mass Spectrom Rev. 2008;27(4):354-77.

29. Baerenfaller K, Grossmann J, Grobei MA, Hull R, Hirsch-Hoffmann M, Yalovsky S, Zimmermann P, Grossniklaus U, Gruissem W, Baginsky S. Genome-scale proteomics reveals Arabidopsis thaliana gene models and proteome dynamics. Science. 2008;320(5878):938-41.

30. Abdallah C, Dumas-Gaudot E, Renaut J, Sergeant K. Gel-based and Gelfree quantitative proteomics approaches at a glance. Int J Plant Genomics. 2012:2012:494572.

31. Fang Y, Robinson DP, Foster LJ. Quantitative analysis of proteome coverage and recovery rates for upstream fractionation methods in proteomics. J Proteome Res. 2010;9(4):1902-12.

32. Piersma SR, Fiedler U, Span S, Lingnau A, Pham TV, Hoffmann S, Kubbutat $M H G$, Jiménez CR. Workflow comparison for label-free, quantitative secretome proteomics for cancer biomarker discovery: method evaluation, differential analysis, and verification in serum. J Proteome Res. 2010;9(4):1913-22.

33. Abraham P, Adams R, Giannone RJ, Kalluri U, Ranjan P, Erickson B, Shah M, Tuskan GA, Hettich RL. Defining the boundaries and characterizing the landscape of functional genome expression in vascular tissues of Populus using shotgun proteomics. J Proteome Res. 2012;11(1):449-60.

34. Picotti $P$, Bodenmiller B, Mueller LN, Domon B, Aebersold R. Full dynamic range proteome analysis of $S$. Cerevisiae by targeted proteomics. Cell. 2009;138(4):795-806

35. Abraham P, Giannone RJ, Adams RM, Kalluri U, Tuskan GA, Hettich RL. Putting the pieces together: high-performance LC-MS/MS provides network-, pathway-, and protein-level perspectives in Populus. Mol Cell Proteomics. 2013;12(1):106-19. 
36. Bailey-Serres J, Sorenson R, Juntawong P. Getting the message across: cytoplasmic ribonucleoprotein complexes. Trends Plant Sci. 2009;14(8):443-53.

37. Marondedze C, Thomas L, Gehring C, Lilley KS. Changes in the Arabidopsis RNA-binding proteome reveal novel stress response mechanisms. BMC Plant Biol. 2019;19(1):1-11.

38. Reichel M, Liao Y, Rettel M, Ragan C, Evers M, Alleaume AM, Horos R, Hentze MW, Preiss T, Millar AA. In planta determination of the mRNAbinding proteome of Arabidopsis etiolated seedlings. Plant Cell. 2016;28(10):2435-52.

39. Tuan PA, Kumar R, Rehal PK, Toora PK, Ayele BT. Molecular mechanisms underlying abscisic acid/gibberellin balance in the control of seed dormancy and germination in cereals. Front Plant Sci. 2018;9:668.

40. Parihar D, Bameta A, Kumari A, Gaur AK. Metabolite profiling of in-vitro established cultures of Picrorhiza kurroa Royle Ex Benth. in different growth regime (S). Int J Pure App Biosci. 2018;6(2):1663-71.

41. Huber SC. Exploring the role of protein phosphorylation in plants: from signalling to metabolism. Biochem Soc Trans. 2007;35(1):28-32.

42. Briggs AG, Bent AF. Poly (ADP-Ribosylation) in plants. Trends Plant Sci. 2011;16(7):372-80.

43. Debeaujon I, Peeters AJ, Léon-Kloosterziel KM, Koornneef M. The transparent testa 12 gene of Arabidopsis encodes a multidrug secondary transporter-like protein required for flavonoid sequestration in vacuoles of the seed coat endothelium. Plant Cell. 2001;13(4):853-71.

44. Pei H, Ma N, Tian J, Luo J, Chen J, Li J, Zheng Y, Chen X, Fei Z, Gao J. An NAC transcription factor controls ethylene-regulated cell expansion in flower petals. Plant Physiol. 2013;163(2):775-91.

45. Ezura K, Ji-Seong K, Mori K, Suzuki Y, Kuhara S, Ariizumi T, Ezura H. Genome-wide identification of pistil-specific genes expressed during fruit set initiation in tomato (Solanum lycopersicum). PLoS One. 2017;12(7):e0180003.

46. Jain M, Nijhawan A, Arora R, Agarwal P, Ray S, Sharma P, Kapoor S, Tyagi AK, Khurana JP. F-box proteins in rice. Genome-wide analysis, classification, temporal and spatial gene expression during panicle and seed development, and regulation by light and abiotic stress. Plant Physiol. 2007;143(4):1467-83.

47. Bao Y, Kost B, Chua N. Reduced expression of alpha-tubulin genes in Arabidopsis thaliana specifically affects root growth and morphology, root hair development and root gravitropism. Plant J. 2001;28(2):145-57.

48. Anders N, Nielsen M, Keicher J, Stierhof YD, Furutani M, Tasaka M, Skriver K, Jürgens G. Membrane association of the Arabidopsis ARF exchange factor GNOM involves interaction of conserved domains. Plant Cell. 2008;20(1):142-51.

49. Taylor MR, Reinders A, Ward JM. Transport function of rice amino acid permeases (AAPs). Plant Cell Physiol. 2015;56(7):1355-63.

50. Reintanz B, Szyroki A, Ivashikina N, Ache P, Godde M, Becker D, Palme K, Hedrich R. AtKC1, a silent Arabidopsis potassium channel a-subunit modulates root hair $\mathrm{K}^{+}$influx. Proc Natl Acad Sci. 2002;99(6):4079-84.

51. Ishibashi T, Kimura S, Furukawa T, Hatanaka M, Hashimoto J, Sakaguchi K. Two types of replication protein A 70 kDa subunit in rice, Oryza sativa: molecular cloning, characterization, and cellular \& tissue distribution. Gene. 2001;272(1-2):335-43.

52. Yu FH, Wang N, He WM, Chu Y, Dong M. Adaptation of rhizome connections in drylands: increasing tolerance of clones to wind erosion. Ann Bot 2008;102(4):571-7.

53. Xia J, Yamaji N, Che J, Shen RF, Ma JF. Normal root elongation requires arginine produced by argininosuccinate lyase in rice. Plant J. 2014;78(2):215-26.

54. Ryder N, Dorn KM, Huitsing M, Adams M, Ploegstra J, DeHaan L, Larson S, Tintle NL. Transcriptome assembly and annotation of johnsongrass (Sorghum halepense) rhizomes identify candidate rhizome-specific genes. Plant Direct. 2018;2(6):e00065.

55. Schnable PS, Hochholdinger F, Nakazono M. Global expression profiling applied to plant development. Curr Opin Plant Biol. 2004;7(1):50-6.

56. Toddl GW. Photosynthesis and respiration of vegetative and reproductive parts of wheat and barley plants in response to increasing temperature. In: Proceedings of the Oklahoma Academy of Science. 1982. p. 57-62.

57. Carmo-Silva E, Scales JC, Madgwick PJ, Parry MAJ. Optimizing Rubisco and its regulation for greater resource use efficiency. Plant Cell Environ. 2015;38(9):1817-32
58. Huang W, Hu H, Zhang SB. Photorespiration plays an important role in the regulation of photosynthetic electron flow under fluctuating light in tobacco plants grown under full sunlight. Front Plant Sci. 2015;6:621.

59. Hou Q, Ufer G, Bartels D. Lipid signalling in plant responses to abiotic stress. Plant Cell Environ. 2016:39(5):1029-48.

60. Ruan YL, Patrick JW, Bouzayen M, Osorio S, Fernie AR. Molecular regulation of seed and fruit set. Trends Plant Sci. 2012;17(11):656-65.

61. Ruan YL. Sucrose metabolism: gateway to diverse carbon use and sugar signaling. Annu Rev Plant Biol. 2014;65:33-67.

62. Lata C, Yadav A, Prasad M. Role of plant transcription factors in abiotic stress tolerance. Abiotic Stress Response in Plants, INTECH Open Access Publishers. 2011;10:269-96.

63. Munemasa S, Hauser F, Park J, Waadt R, Brandt B, Schroeder Jl. Mechanisms of abscisic acid-mediated control of stomatal aperture. Curr Opin Plant Biol. 2015;28:154-62.

64. Jogawat A, Yadav B, Narayan OP. Metal transporters in organelles and their roles in heavy metal transportation and sequestration mechanisms in plants. Physiol Plant. 2021;173:259-75.

65. Venkataraman K. India's Biodiversity Act 2002 and its role in conservation. Trop Ecol. 2009;50(1):23.

66. Kumar R, Joshi R, Kumari M, Thakur R, Kumar D, Kumar S. Elevated $\mathrm{CO}_{2}$ and temperature influence key proteins and metabolites associated with photosynthesis, antioxidant and carbon metabolism in Picrorhiza kurroa. J Proteomics. 2020;219:103755.

67. Bradford MM. A Rapid and sensitive method for the quantitation of microgram quantities of protein utilizing the principle of protein-dye binding. Anal Biochem. 1976;72(1-2):248-54.

68. Shevchenko A, Jensen ON, Podtelejnikov AV, Sagliocco F, Wilm M, Vorm O, Mortensen P, Shevchenko A, Boucherie H, Mann M. Linking genome and proteome by mass spectrometry: large-scale identification of yeast proteins from two dimensional gels. Proc Natl Acad Sci. 1996;93(25):14440-5.

69. Schwanhäusser B, Busse D, Li N, Dittmar G, Schuchhardt J, Wolf J, Chen W, Selbach M. Global quantification of mammalian gene expression control. Nature. 2011;473(7347):337-42.

70. Zhang C, Chen Y, Mao X, Huang Y, Jung SY, Jain A, Qin J, Wang Y. A bioinformatic algorithm for analyzing cell signaling using temporal proteomic data. Proteomics. 2017;17(22):1600425.

71. Krzywinski M, Schein J, Birol I, Connors J, Gascoyne R, Horsman D, Jones SJ, Marra MA. Circos: An information aesthetic for comparative genomics. Genome Res. 2009;19(9):1639-45.

72. Tian T, Liu Y, Yan H, You Q, Yi X, Du Z, Xu W, Su Z. AgriGO v2.0: A GO analysis toolkit for the agricultural community, 2017 update. Nucleic Acids Res. 2017;45(W1):W122-9.

73. Darzi Y, Letunic I, Bork P, Yamada T. iPath3.0: Interactive pathways explorer V3. Nucleic Acids Res. 2018;46(W1):W510-3.

74. Ge SX, Jung D, Yao R. Shiny GO: A graphical gene-set enrichment tool for animals and plants. Bioinformatics. 2020;36(8):2628-9.

75. Pejaver V, Hsu W, Xin F, Dunker AK, Uversky VN, Radivojac P. The structural and functional signatures of proteins that undergo multiple events of post-translational modification. Protein Sci. 2014;23(8):1077-93.

76. El-Gebali S, Mistry J, Bateman A, Eddy SR, Luciani A, Potter SC, Qureshi M, Richardson LJ, Salazar GA, Smart A. The Pfam protein families database in 2019. Nucleic Acids Res. 2019:47(D1):D427-32

77. Kozlowski LP. IPC-isoelectric point calculator. Biol Direct. 2016;11(1):55

78. Stothard P. The sequence manipulation suite: javascript programs for analyzing and formatting protein and dna sequences. Biotechniques. 2000;28(6):1102-4

79. Mészáros B, Erdős G, Dosztányi Z. IUPred2A: Context-dependent prediction of protein disorder as a function of redox state and protein binding Nucleic Acids Res. 2018;46(W1):W329-37.

80. Bailey TL. DREME: motif discovery in transcription factor ChIP-Seq data. Bioinformatics. 2011;27(12):1653-9.

\section{Publisher's Note}

Springer Nature remains neutral with regard to jurisdictional claims in published maps and institutional affiliations. 